\title{
Ligand-Dependent Scope and Divergent Mechanistic Behavior in Nickel-Catalyzed Reductive Couplings of Aldehydes and Alkynes.
}

\author{
Gireesh M. Mahandru, Gang Liu, and John Montgomery* \\ Department of Chemistry, Wayne State University, Detroit, MI 48202.
}

\section{Supporting Information}

All reagents were used as received unless otherwise noted. Tetrahydrofuran (THF) was treated under nitrogen using a solvent purification system (Innovative Technology, Inc., Model \# SPS-400-3). All aldehydes and alkynes were freshly distilled prior to use. $\mathrm{Ni}(\mathrm{COD})_{2}$ and 1,3-bis(2,4,6-trimethyl-phenyl)imidazolium chloride (Strem Chemicals, Inc., used as received) were stored and weighed in an inert atmosphere glovebox. All reactions were conducted in flame-dried glassware under an oxygen-free atmosphere of argon or nitrogen. ${ }^{1} \mathrm{H}$ and ${ }^{13} \mathrm{C}$ spectra were obtained in $\mathrm{CDCl}_{3}$, unless otherwise noted, on a Varian Mercury 400, or Varian Unity $500 \mathrm{MHz}$ instrument. Chemical shifts of ${ }^{1} \mathrm{H}$ NMR spectra were recorded in parts per million (ppm) on the $\delta$ scale from an internal standard

of residual chloroform (7.27 ppm). Chemical shifts of ${ }^{13} \mathrm{C}$ NMR spectra are reported in ppm from the central peak of $\mathrm{CDCl}_{3}(77.0 \mathrm{ppm})$ on the $\delta$ scale. High Resolution mass spectra (HRMS) were obtained on a Kratos MS 80 mass spectrometer by the Central Instrumentation Facility, Department of Chemistry, Wayne State University, Detroit, Michigan.

General Procedure A for the $\mathrm{Ni(COD)})_{2} /$ carbene catalyzed couplings of aldehydes and alkynes. (Table 1. Examples 1 - 9.)

An $8 \mathrm{~mL}$ THF solution of $\mathrm{Ni}(\mathrm{COD})_{2}(28 \mathrm{mg}, 0.1 \mathrm{mmol})$ and the imidazolium salt 1 (34 mg, $0.1 \mathrm{mmol})$ was prepared. The mixture was cooled to $0^{\circ} \mathrm{C}$, and $n \mathrm{BuLi}(1.6 \mathrm{M} / \mathrm{hex}, 62$ 
$\mu \mathrm{L}, 0.1 \mathrm{mmol}$ ) was added dropwise. The mixture initially turned green and on standing at $0^{\circ} \mathrm{C}$ for $50 \mathrm{~min}$ had a deep red color. Triethylsilane $(320 \mu \mathrm{L}, 2.0 \mathrm{mmol})$ was then added dropwise and the mixture was heated to $45^{\circ} \mathrm{C}$ for $5 \mathrm{~min}$. At the same temperature, the aldehyde $(1.0 \mathrm{mmol})$ was added dropwise followed by addition of the alkyne $(1.2 \mathrm{mmol})$ in $2 \mathrm{~mL}$ THF over 15 min using a syringe pump. After addition of the alkyne was complete, the mixture was stirred for 15 min at $45{ }^{\circ} \mathrm{C}$, followed by quenching with aqueous sat. solution of sodium bicarbonate and extracting $3 \mathrm{x}$ with ethyl acetate. $\mathrm{MgSO}_{4}$ was then added, the solution was filtered and the solvent was removed using a rotary evaporator. The crude reaction mixture was purified by column chromatography $\left(\mathrm{SiO}_{2}\right.$, hexanes, unless otherwise noted), and the protected allyl alcohols were isolated as colorless to pale yellow oils.

\section{General Procedure B for the $\mathrm{Ni}(\mathrm{COD})_{2} /$ carbene catalyzed couplings of aldehydes} and alkynes. ( Table 1. Example 10. )

A $5 \mathrm{~mL}$ solution of the imidazolium salt $1(34 \mathrm{mg}, 0.1 \mathrm{mmol})$ in THF was prepared. This solution was then cooled to $0{ }^{\circ} \mathrm{C}$ and $n \mathrm{BuLi}(1.6 \mathrm{M} / \mathrm{hex}, 62 \mu \mathrm{L}, 0.1 \mathrm{mmol})$ was added dropwise. This mixture was kept at the same temperature for 10 minutes during which the yellow solution went to colorless. $\mathrm{Ni}(\mathrm{COD})_{2}(28 \mathrm{mg}, 0.1 \mathrm{mmol})$, dissolved in $3 \mathrm{~mL}$ THF was then added by cannula and the solution was stirred at $0{ }^{\circ} \mathrm{C}$ for $10 \mathrm{~min}$. Triethylsilane (320 $\mu \mathrm{L}, 2.0 \mathrm{mmol})$ was then added followed by addition of the aldehyde $(1.0 \mathrm{mmol}$ ). The alkyne $(1.5 \mathrm{mmol})$ in $2 \mathrm{~mL}$ THF was then added over 15 minutes by syringe pump. The mixture was then allowed to warm to $\mathrm{rt}$ and was stirred overnight. The reaction mixture was quenched with a aqueous sat. solution of sodium bicarbonate and extracted 3x with ethyl acetate. $\mathrm{MgSO}_{4}$ was then added, the solution was filtered, and the solvent 
removed by rotary evaporation. The crude reaction mixture was purified by column chromatography $\left(\mathrm{SiO}_{2}\right.$, hexanes, unless otherwise noted), and the protected allyl alcohols were isolated as colorless to pale yellow oils.<smiles>COC(C(C)=Cc1ccccc1)c1ccccc1</smiles>

\section{Table 1, Entry 1, Triethyl-(2-methyl-1,3-diphenyl-allyloxy)-silane}

Following the general procedure $\mathrm{A}, \mathrm{Ni}(\mathrm{COD})_{2}(28 \mathrm{mg}, 0.1 \mathrm{mmol})$, imidazolium salt 1 (34 $\mathrm{mg}, 0.1 \mathrm{mmol}), \mathrm{nBuLi}(1.6 \mathrm{M} / \mathrm{hexanes}, 62 \mu \mathrm{L}, 0.1 \mathrm{mmol})$, triethylsilane $(320 \mu \mathrm{L}, 2.0$ mmol), 1-phenyl-propyne (139 mg, $1.2 \mathrm{mmol})$, and benzaldeyhde (102 $\mu \mathrm{L}, 1.0 \mathrm{mmol})$ were employed to give triethyl-(2-methyl-1,3-diphenyl-allyloxy)-silane (283 mg, 0.84 mmol, $84 \%,>98: 2$ mixture of regioisomers), after column chromatography $\left(\mathrm{SiO}_{2}\right.$, hexanes) as a colorless oil. ${ }^{1} \mathrm{H} \mathrm{NMR}\left(500 \mathrm{MHz}, \mathrm{CDCl}_{3}\right) \delta 7.56(\mathrm{~d}, J=7.5 \mathrm{~Hz}, 2 \mathrm{H}) 7.41$ $7.45(\mathrm{~m}, 6 \mathrm{H}) 7.30-7.36(\mathrm{~m}, 2 \mathrm{H}) 6.85(\mathrm{~s}, 1 \mathrm{H}), 5.38(\mathrm{~s}, 1 \mathrm{H}) 1.80(\mathrm{~d}, J=1.5 \mathrm{~Hz}, 3 \mathrm{H}) 1.09(\mathrm{t}$, $J=8.5 \mathrm{~Hz}, 9 \mathrm{H}) 0.78(\mathrm{q}, J=8.0 \mathrm{~Hz}, 6 \mathrm{H}) ;{ }^{13} \mathrm{C}\left(125 \mathrm{MHz}, \mathrm{CDCl}_{3}\right) \delta 143.7,141.2,138.2$, 129.3, 128.4, 128.3, 127.2, 126.7, 126.5, 125.9, 80.3, 13.5, 7.2, 5.3. IR (film, $\mathrm{cm}^{-1}$ ) 2954.4, 2875.0, 1492.0, 1088.1, 1064.7. HRMS (EI) $\mathrm{m} / \mathrm{z}$ calculated for $\mathrm{C}_{22} \mathrm{H}_{30} \mathrm{OSi}$ 338.2066, found 338.2070 $\left(\mathrm{M}^{+}\right)$.

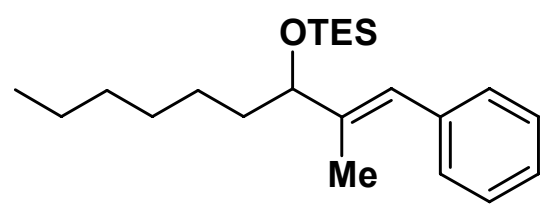

\section{Table 1: Entry 2, Triethyl-(1-hexyl-2-methyl-3-phenyl-allyloxy)-silane}

Following the general procedure $\mathrm{A}, \mathrm{Ni}(\mathrm{COD})_{2}(28 \mathrm{mg}, 0.1 \mathrm{mmol})$, imidazolium salt 1 (34 $\mathrm{mg}, 0.1 \mathrm{mmol}), \mathrm{nBuLi}(1.6 \mathrm{M} / \mathrm{h}$ exanes, $62 \mu \mathrm{L}, 0.1 \mathrm{mmol})$, triethylsilane $(320 \mu \mathrm{L}, 2.0$ 
mmol), 1-phenyl-propyne (139 mg, $1.2 \mathrm{mmol})$, and heptaldehyde (139 $\mu \mathrm{L}, 1.0 \mathrm{mmol})$ were employed to give triethyl-(1-hexyl-2-methyl-3-phenyl-allyloxy)-silane (284 mg, $0.82 \mathrm{mmol}, 82 \%,>98: 2$ mixture of regioisomers) after column chromatography $\left(\mathrm{SiO}_{2}\right.$, hexanes) as a colorless oil. ${ }^{1} \mathrm{H}$ NMR $\left(500 \mathrm{MHz}, \mathrm{CDCl}_{3}\right) \delta 7.40(\mathrm{t}, J=8.0 \mathrm{~Hz}, 2 \mathrm{H}) 7.35$ (d, $J=7.0 \mathrm{~Hz}, 2 \mathrm{H}) 7.26-7.29(\mathrm{~m}, 1 \mathrm{H}) 6.51(\mathrm{~s}, 1 \mathrm{H}) 5.86(\mathrm{q}, J=7.5 \mathrm{~Hz}, 0.02 \mathrm{H}$, minor regioisomer) 4.39 (t, J=6.5 Hz, $0.02 \mathrm{H}$, minor regioisomer) $4.21(\mathrm{t}, J=6.0 \mathrm{~Hz}, 1 \mathrm{H}) 1.92$ (d, $J=1.5 \mathrm{~Hz}, 3 \mathrm{H}) 1.62-1.74(\mathrm{~m}, 2 \mathrm{H}) 1.29-1.53(\mathrm{~m}, 8 \mathrm{H}) 1.07(\mathrm{t}, J=8.0 \mathrm{~Hz}, 9 \mathrm{H}) 0.99(\mathrm{t}$, $J=7.0 \mathrm{~Hz}, 3 \mathrm{H}) 0.72(\mathrm{q}, J=8.0 \mathrm{~Hz}, 6 \mathrm{H}) ;{ }^{13} \mathrm{C}\left(125 \mathrm{MHz}, \mathrm{CDCl}_{3}\right) \delta 141.4,138.3,129.2$, $128.3,126.4,125.3,79.1,36.8,32.2,29.7,26.1,23.0,14.4,13.3,7.2,5.2$. IR (film, $\mathrm{cm}^{-1}$ ) 2954.8, 2931.0, 1457.5, 1238.1, 1075.0, 1004.9, 745.3. HRMS (EI) $\mathrm{m} / z$ calculated for $\mathrm{C}_{22} \mathrm{H}_{38} \mathrm{OSi} 346.2692$, found $346.2698\left(\mathrm{M}^{+}\right)$.

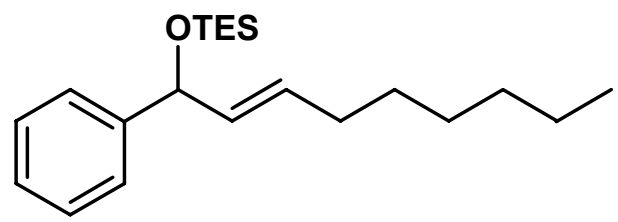

Table 1: Entry 3, Triethyl-(1-phenyl-non-2-enyloxy)-silane

Following the general procedure $\mathrm{A}, \mathrm{Ni}(\mathrm{COD})_{2}(28 \mathrm{mg}, 0.1 \mathrm{mmol})$, imidazolium salt 1 (34 $\mathrm{mg}, 0.1 \mathrm{mmol}), \mathrm{nBuLi}(1.6 \mathrm{M} / \mathrm{hexanes}, 62 \mu \mathrm{L}, 0.1 \mathrm{mmol}$ ), triethylsilane ( $320 \mu \mathrm{L}, 2.0$ mmol), 1-octyne (132 mg, $1.2 \mathrm{mmol})$ and benzaldehyde $(102 \mu \mathrm{L}, 1.0 \mathrm{mmol})$ were employed to give triethyl-(1-phenyl-non-2-enyloxy)-silane (236 mg, $0.71 \mathrm{mmol}, 71 \%$, $>98: 2$ mixture of regioisomers) after column chromatography $\left(\mathrm{SiO}_{2}\right.$, hexanes) as a colorless oil. ${ }^{1} \mathrm{H}$ NMR $\left(400 \mathrm{MHz}, \mathrm{CDCl}_{3}\right) \delta 7.36(\mathrm{dt}, J=14.8,7.2 \mathrm{~Hz}, 4 \mathrm{H})$ 7.22-7.26 (m, 1H) $5.69(\mathrm{dt}, J=15.6,6.4 \mathrm{~Hz}, 1 \mathrm{H}) 5.58(\mathrm{dd}, J=6.8,15.6 \mathrm{~Hz}, 1 \mathrm{H}) 5.17(\mathrm{~d}, J=6.4 \mathrm{~Hz}$, $1 \mathrm{H}) 2.10(\mathrm{q}, J=7.2 \mathrm{~Hz}, 2 \mathrm{H}) 1.24-1.47(\mathrm{~m}, 8 \mathrm{H}) 0.97(\mathrm{t}, J=7.2 \mathrm{~Hz}, 9 \mathrm{H}) 0.91(\mathrm{t}, J=6.4 \mathrm{~Hz}$, $3 \mathrm{H}) \quad 0.57-0.71(\mathrm{~m}, 6 \mathrm{H}) ;{ }^{13} \mathrm{C}\left(100 \mathrm{MHz}, \mathrm{CDCl}_{3}\right) \delta 144.8,133.8,131.2,128.3,127.0$, 
126.2, 75.8, 32.4, 32.0, 29.37, 29.16, 22.9, 14.3, 7.1, 5.2. IR ( film, $\mathrm{cm}^{-1}$ ) 2954.8, 2925.3, 1457.9, 1240.5, 1100.9, 1056.3, 1105.9, 965.8 HRMS (EI) $\mathrm{m} / z$ calculated for $\mathrm{C}_{21} \mathrm{H}_{32} \mathrm{OSi}$ 332.2535, found 332.2531 $\left(\mathrm{M}^{+}\right)$.<smiles>COS(=S)(=S)OC(/C=C/c1ccccc1)c1ccccc1</smiles>

Table 1: Entry 4, (1,3-Diphenyl-allyloxy)-triethyl-silane

Following the general procedure $\mathrm{A}, \mathrm{Ni}(\mathrm{COD})_{2}(28 \mathrm{mg}, 0.1 \mathrm{mmol})$, imidazolium salt 1 (34 $\mathrm{mg}, 0.1 \mathrm{mmol}), \mathrm{nBuLi}(1.6 \mathrm{M} / \mathrm{h}$ exanes, $62 \mu \mathrm{L}, 0.1 \mathrm{mmol})$, triethylsilane $(320 \mu \mathrm{L}, 2.0$ mmol), phenyl acetylene (122 mg, $1.2 \mathrm{mmol})$, and benzaldeyhde (102 $\mu \mathrm{L}, 1.0 \mathrm{mmol})$ were employed to give (1,3-diphenyl-allyloxy)-triethyl-silane (234 mg, $0.72 \mathrm{mmol}, 72 \%$, $>98: 2$ mixture of regioisomers) after column chromatography $\left(\mathrm{SiO}_{2}\right.$, hexanes) as a colorless oil. Note: The alkyne was added over $2 \mathrm{~h}$ using a syringe drive. ${ }^{1} \mathrm{H}$ NMR (500 $\left.\mathrm{MHz}, \mathrm{CDCl}_{3}\right) \delta 7.26-7.53(\mathrm{~m}, 10 \mathrm{H}) 6.71(\mathrm{~d}, J=16.0 \mathrm{~Hz}, 1 \mathrm{H}) 6.38(\mathrm{ddd}, J=15.4,6.3,2.5$ $\mathrm{Hz}, 1 \mathrm{H})$ 5.424-5.435 (m, 1H) $1.02-1.06(\mathrm{~m}, 9 \mathrm{H})$ 0.66-0.79 (m, 6H); ${ }^{13} \mathrm{C}(125 \mathrm{MHz}$, $\left.\mathrm{CDCl}_{3}\right) \delta 144.1,137.2,133.5,129.2,128.8,128.6,127.8,127.4,126.8,126.4,75.7,7.1$ 5.3. IR (film, $\mathrm{cm}^{-1}$ ) 2954.5, 2875.1, 1493.6, 1449.3, 1103.6, 1059.1, 1004.6, 965.7, 743.4. HRMS (EI) $m / z$ calculated for $\mathrm{C}_{21} \mathrm{H}_{28} \mathrm{OSi} 324.1909$, found $324.1907\left(\mathrm{M}^{+}\right)$. 


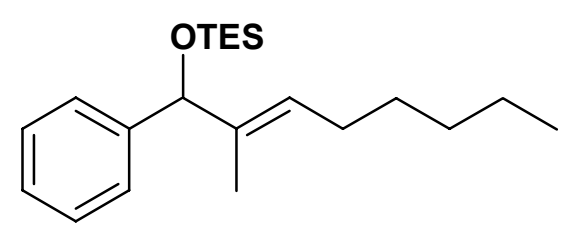

Table 1: Entry 5,

Triethyl-(2-methyl-1-phenyl-oct-2-enyloxy)-silane

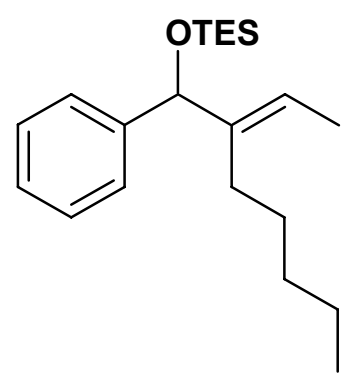

Triethyl-(2-pentyl-1-phenyl-but-2-enyloxy)-sil

Following the general procedure $\mathrm{A}, \mathrm{Ni}(\mathrm{COD})_{2}(28 \mathrm{mg}, 0.1 \mathrm{mmol})$, imidazolium salt 1 (34 $\mathrm{mg}, 0.1 \mathrm{mmol}), \mathrm{nBuLi}(1.6 \mathrm{M} / \mathrm{h}$ exanes, $62 \mu \mathrm{L}, 0.1 \mathrm{mmol})$, triethylsilane $(320 \mu \mathrm{L}, 2.0$ mmol), 2-octyne (132 mg, $1.2 \mathrm{mmol})$, and benzaldeyhde (102 $\mu \mathrm{L}, 1.0 \mathrm{mmol})$ were employed to give a mixture of triethyl-(2-methyl-1-phenyl-oct-2-enyloxy)-silane and triethyl-(2-pentyl-1-phenyl-but-2-enyloxy)-silane (279 mg, $0.84 \mathrm{mmol}, 84 \%$ 1.3:1 mixture of regioisomers) after column chromatography ( $\mathrm{SiO}_{2}$, hexanes) as a colorless oil. Note: The alkyne was added over 15 min using a syringe drive, and the reaction mixture was then heated at $60{ }^{\circ} \mathrm{C}$ for $2 \mathrm{~h} .{ }^{1} \mathrm{H}$ NMR $\left(500 \mathrm{MHz}, \mathrm{CDCl}_{3}\right) \delta 7.40(\mathrm{t}, J=11.0 \mathrm{~Hz}, 2 \mathrm{H})$ $7.32(\mathrm{q}, J=8.0 \mathrm{~Hz}, 2 \mathrm{H}) 7.22-7.25(\mathrm{~m}, 1 \mathrm{H}) 5.67(\mathrm{q}, J=7.5 \mathrm{~Hz}, 0.43 \mathrm{H}) 5.62(\mathrm{t}, J=7.0 \mathrm{~Hz}$, $0.57 \mathrm{H}) 5.15(\mathrm{~s}, 1 \mathrm{H}) 2.03-2.14(\mathrm{~m}, 1.19 \mathrm{H}) 1.87-1.99(\mathrm{~m}, 0.86 \mathrm{H}) 1.68(\mathrm{~d}, J=7.5 \mathrm{~Hz}$, $1.24 \mathrm{H}) 1.45(\mathrm{~m}, 3 \mathrm{H}) 1.35-1.40(\mathrm{~m}, 2.55 \mathrm{H}) 1.17-1.31(\mathrm{~m}, 2.1 \mathrm{H}) 1.05-1.15(\mathrm{~m}, 0.5 \mathrm{H})$ 0.94$1.00(\mathrm{~m}, 10.7 \mathrm{H}) 0.87(\mathrm{t}, J=6.5 \mathrm{~Hz}, 1.36 \mathrm{H}) 0.6-0.69(\mathrm{~m}, 6 \mathrm{H}) ;{ }^{13} \mathrm{C}\left(125 \mathrm{MHz}, \mathrm{CDCl}_{3}\right) \delta$ $144.4,144.1,143.3,138.0,127.99,127.96,126.92,126.89,126.75,126.6,126.2,120.7$, $80.1,79.2,32.5,31.9,29.5,29.0,27.8,27.0,22.9,22.7,14.3,14.2,13.4,11.3,7.11,7.10$ 5.15. IR (film, $\mathrm{cm}^{-1}$ ) 2955.2, 2931.6, 1457.8, 1238.2, 1087.8, 1064.9, 742.5. HRMS (EI) $m / z$ calculated for $\mathrm{C}_{21} \mathrm{H}_{36} \mathrm{OSi} 332.2535$, found $332.2535\left(\mathrm{M}^{+}\right)$. 


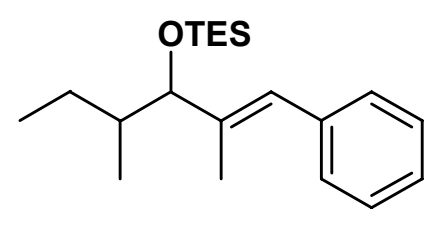

Table 1: Entry 6, (1-sec-Butyl-2-methyl-3-phenyl-allyloxy)-triethyl-silane

Following the general procedure $\mathrm{A}, \mathrm{Ni}(\mathrm{COD})_{2}(28 \mathrm{mg}, 0.1 \mathrm{mmol})$, imidazolium salt 1 (34 $\mathrm{mg}, 0.1 \mathrm{mmol}), \mathrm{nBuLi}(1.6 \mathrm{M} / \mathrm{h}$ exanes, $62 \mu \mathrm{L}, 0.1 \mathrm{mmol})$, triethylsilane $(320 \mu \mathrm{L}, 2.0$ mmol), 1-phenyl propyne (139 mg, $1.2 \mathrm{mmol}$ ), and 2-methylbutyraldehyde (107 $\mu \mathrm{L}, 1.0$ mmol) were employed to give (1-sec-butyl-2-methyl-3-phenyl-allyloxy)-triethyl-silane (256 mg, $0.81 \mathrm{mmol}, 81 \%, 3: 2 \mathrm{dr},>98: 2$ mixture of regioisomers) after column chromatography $\left(\mathrm{SiO}_{2}\right.$, hexanes) as a colorless oil. ${ }^{1} \mathrm{H} \mathrm{NMR}\left(400 \mathrm{MHz}, \mathrm{CDCl}_{3}\right) \delta 7.36(\mathrm{t}$, $J=8.4 \mathrm{~Hz}, 2 \mathrm{H}) 7.29(\mathrm{~d}, J=8.0 \mathrm{~Hz}, 2 \mathrm{H}) 7.21-7.26(\mathrm{~m}, 1 \mathrm{H}) 6.42(\mathrm{~s}, 0.6 \mathrm{H}) 6.39(\mathrm{~s}, 0.4 \mathrm{H})$ $3.85(\mathrm{~d}, J=7.6 \mathrm{~Hz}, 0.6 \mathrm{H}) 3.79(\mathrm{~d}, J=8.4 \mathrm{~Hz}, 0.4 \mathrm{H}) 1.83(\mathrm{~s}, 3 \mathrm{H}) 1.56-1.62(\mathrm{~m}, 1 \mathrm{H}) 1.38-$ $1.47(\mathrm{~m}, 0.7 \mathrm{H}) 1.03-1.17(\mathrm{~m}, 1 \mathrm{H}) 0.90-1.01(\mathrm{~m}, 14 \mathrm{H}) 0.80(\mathrm{~d}, J=7.2 \mathrm{~Hz}, 1.3 \mathrm{H}) 0.65(\mathrm{q}$, $J=8.0 \mathrm{~Hz}, 6 \mathrm{H}) ;{ }^{13} \mathrm{C}\left(125 \mathrm{MHz}, \mathrm{CDCl}_{3}\right) \delta 140.56,140.45,138.30,138.22,129.1,128.3$, $126.8,126.43,126.38,126.36,84.0,83.2,39.1,38.9,26.3,25.3,15.7,14.9,13.9,13.3$, 12.0, 11.7, 7.2, 5.22, 5.20 IR (film, $\mathrm{cm}^{-1}$ ) 2957.8, 2875.5, 1458.0, 1064.8, 1006.5 HRMS (EI) $m / z$ calculated for $\mathrm{C}_{19} \mathrm{H}_{31} \mathrm{OSi} 303.2144$, found $303.2143\left(\mathrm{M}^{+}\right)$.<smiles>[CH]OC(c1ccc(OC)cc1)C(OC)/C(C)=C/c1ccccc1</smiles>

Table 1: Entry 7, Triethyl-[1-(4-methoxy-phenyl)-2-methyl-3-phenyl-allyloxy]-silane Following the general procedure $\mathrm{A}, \mathrm{Ni}(\mathrm{COD})_{2}(28 \mathrm{mg}, 0.1 \mathrm{mmol})$, imidazolium salt 1 (34 $\mathrm{mg}, 0.1 \mathrm{mmol}), \mathrm{nBuLi}(1.6 \mathrm{M} /$ hexanes, $62 \mu \mathrm{L}, 0.1 \mathrm{mmol})$, triethylsilane $(320 \mu \mathrm{L}, 2.0$ mmol), 1-phenyl propyne (139 mg, $1.2 \mathrm{mmol})$, and $p$-methoxybenzaldehyde (122 $\mu \mathrm{L}, 1.0$ 
mmol) were employed to give triethyl-[1-(4-methoxy-phenyl)-2-methyl-3-phenylallyloxy]-silane (242 mg, $0.66 \mathrm{mmol}, 66 \%,>98: 2$ mixture of regioisomers) after silica gel chromatography $\left(10: 1 \mathrm{Hex} /\right.$ EtOAc) as a pale yellow oil. ${ }^{1} \mathrm{H} \mathrm{NMR}\left(400 \mathrm{MHz}, \mathrm{CDCl}_{3}\right)$ 7.31-7.37 (m, 6H) 7.21-7.26 (m, 1H) 6.88-6.90 (m, 2H) $6.73(\mathrm{~s}, 1 \mathrm{H}) 5.22(\mathrm{~s}, 1 \mathrm{H}) 3.83(\mathrm{~s}$, 3H) $1.70(\mathrm{~s}, 3 \mathrm{H}) 0.98(\mathrm{t}, J=8.0 \mathrm{~Hz}, 9 \mathrm{H}) 0.67(\mathrm{q}, J=8.4 \mathrm{~Hz}, 6 \mathrm{H}) ;{ }^{13} \mathrm{C}\left(125 \mathrm{MHz}, \mathrm{CDCl}_{3}\right)$ $\delta 158.8,141.2,138.2,135.8,129.2,128.3,127.6,126.5,125.4,113.6,79.7,55.5,13.6$ 7.1, 5.2. IR (film, $\mathrm{cm}^{-1}$ ) 2952.8, 1508.8, 1246.6, 1167.9, 1075.8 HRMS (EI) $\mathrm{m} / \mathrm{z}$ calculated for $\mathrm{C}_{23} \mathrm{H}_{32} \mathrm{OSi} 368.2172$, found $368.2170\left(\mathrm{M}^{+}\right)$.<smiles>C=C(C)C=C(c1ccccc1)C(OC#[SH])c1ccccc1</smiles>

Table 1: Entry 8, Triethyl-(4-methyl-1,2-diphenyl-penta-2,4-dienyloxy)-silane

Following the general procedure $\mathrm{A}, \mathrm{Ni}(\mathrm{COD})_{2}(28 \mathrm{mg}, 0.1 \mathrm{mmol})$, imidazolium salt 1 (34 $\mathrm{mg}, 0.1 \mathrm{mmol}), \mathrm{nBuLi}(1.6 \mathrm{M} /$ hexanes, $62 \mu \mathrm{L}, 0.1 \mathrm{mmol})$, triethylsilane $(320 \mu \mathrm{L}, 2.0$ mmol), (3-methyl-but-3-en-1-ynyl)-benzene (170 mg, $1.2 \mathrm{mmol})$, and benzaldehyde (102 $\mu \mathrm{L}, 1.0 \mathrm{mmol})$ were employed to give triethyl-(4-methyl-1,2-diphenyl-penta-2,4dienyloxy)-silane (305 mg, $0.84 \mathrm{mmol}, 84 \%,>98: 2$ mixture of regioisomers) after silica gel chromatography (hexanes) as a colorless oil. ${ }^{1} \mathrm{H} \mathrm{NMR}\left(500 \mathrm{MHz}, \mathrm{CDCl}_{3}\right)$ 7.14-7.26 (m, 8H) $6.84(\mathrm{~d}, J=7.5 \mathrm{~Hz}, 2 \mathrm{H}) 6.59(\mathrm{~s}, 1 \mathrm{H}) 5.33(\mathrm{~s}, 1 \mathrm{H}) 4.94(\mathrm{~s}, 1 \mathrm{H}) 4.89(\mathrm{~s}, 1 \mathrm{H}) 1.37$ (s, 3H) $0.95(\mathrm{t}, J=8.0 \mathrm{~Hz}, 9 \mathrm{H}) 0.634(\mathrm{q}, J=8.0 \mathrm{~Hz}, 6 \mathrm{H}) ;{ }^{13} \mathrm{C}\left(125 \mathrm{MHz}, \mathrm{CDCl}_{3}\right) \delta 144.3$, $143.1,142.3,139.1,130.1,128.5,127.9,127.4,127.16,127.10,126.90,118.2,79.9,22.5$, 
7.1, 5.1 IR (film, $\mathrm{cm}^{-1}$ ) 2956.2, 2875.4, 1492.0, 1452.0, 1101.4, 1006.4 HRMS (EI) $\mathrm{m} / \mathrm{z}$ calculated for $\mathrm{C}_{24} \mathrm{H}_{32} \mathrm{OSi} 364.2222$, found $364.2225\left(\mathrm{M}^{+}\right)$.

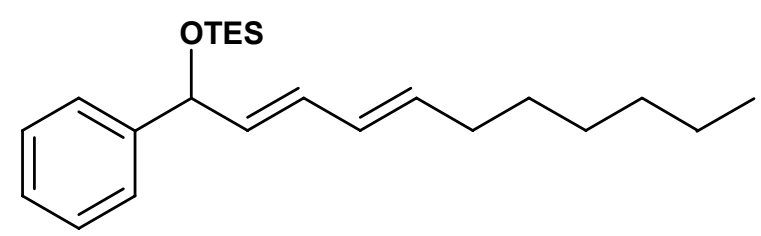

Table 1: Entry 9, Triethyl-(1-phenyl-undeca-2,4-dienyloxy)-silane

Following the general procedure $\mathrm{A}, \mathrm{Ni}(\mathrm{COD})_{2}(15 \mathrm{mg}, 0.055 \mathrm{mmol})$, imidazolium salt 1 (34 mg, $0.55 \mathrm{mmol}), \mathrm{nBuLi}(1.6 \mathrm{M} / \mathrm{hexanes}, 62 \mu \mathrm{L}, 0.055 \mathrm{mmol})$, triethylsilane (166 $\mu \mathrm{L}$, $1.102 \mathrm{mmol})$, dec-3-en-1-yne (90 mg, $0.662 \mathrm{mmol})$, and benzaldehyde $(56 \mu \mathrm{L}, 0.55$ mmol) were employed to give triethyl-(1-phenyl-undeca-2,4-dienyloxy)-silane (111 mg, $0.31 \mathrm{mmol}, 56 \%,>98: 2$ mixture of regioisomers) after silica gel chromatography (hexanes) as a pale yellow oil. ${ }^{1} \mathrm{H}$ NMR $\left(400 \mathrm{MHz}, \mathrm{C}_{6} \mathrm{D}_{6}\right) \delta$ 7.29-7.36 (m, 4H) 7.22 (tt, $J$ $=6.8,2.4 \mathrm{~Hz}, 1 \mathrm{H}) 6.18(\mathrm{dd}, J=14.8,10.4 \mathrm{~Hz}, 1 \mathrm{H}) 6.00(\mathrm{dd}, J=15.0,9.6 \mathrm{~Hz}, 1 \mathrm{H}) 5.63-$ $5.72(\mathrm{~m}, 2 \mathrm{H}) 5.19(\mathrm{~d}, J=6.4 \mathrm{~Hz}, 1 \mathrm{H}) 2.06(\mathrm{q}, J=7.2 \mathrm{~Hz}, 2 \mathrm{H}) 1.27-1.39(\mathrm{~m}, 9 \mathrm{H}) 0.87-$ $0.95(\mathrm{~m}, 12 \mathrm{H}) \quad 0.53-0.67(\mathrm{~m}, 6 \mathrm{H}) ;{ }^{13} \mathrm{C}\left(125 \mathrm{MHz}, \mathrm{CDCl}_{3}\right) \delta 144.4,135.4,134.4,129.88$, 129.77, 128.4, 127.2, 126.2, 75.4, 32.9, 32.0, 29.4, 29.1, 22.8, 14.3, 7.0, 5.2. IR (film, $\mathrm{cm}^{-}$ 1) 2956.6, 2920.2, 1452.4, 1098.8, 1061.9, 987.6, 742.3, 698.3. HRMS (EI) $\mathrm{m} / \mathrm{z}$ calculated for $\mathrm{C}_{23} \mathrm{H}_{38} \mathrm{OSi} 358.2692$, found $358.2690\left(\mathrm{M}^{+}\right)$.<smiles>CCOC(C=CCCCCO)c1ccccc1</smiles>

Table 1: Entry 10, 7-Phenyl-7-triethylsilanyloxy-hept-5-en-1-ol 
Following the general procedure $\mathrm{B}, \mathrm{Ni}(\mathrm{COD})_{2}(28 \mathrm{mg}, 0.1 \mathrm{mmol})$, imidazolium salt 1 (34 $\mathrm{mg}, 0.1 \mathrm{mmol}), \mathrm{nBuLi}(1.6 \mathrm{M} / \mathrm{h}$ exanes, $62 \mu \mathrm{L}, 0.1 \mathrm{mmol})$, triethylsilane $(320 \mu \mathrm{L}, 2.0$ mmol), 5-hexyn-1-ol (147 mg, $1.5 \mathrm{mmol})$, and benzaldehyde (102 $\mu \mathrm{L}, 1.0 \mathrm{mmol})$ were employed to give 7-phenyl-7-triethylsilanyloxy-hept-5-en-1-ol (229 mg, $0.72 \mathrm{mmol}, 72$ $\%,>98: 2$ mixture of regioisomers) after silica gel chromatography (3:1 Hex/ EtOAc) as a pale yellow oil. ${ }^{1} \mathrm{H}$ NMR $\left(500 \mathrm{MHz}, \mathrm{CDCl}_{3}\right) \delta$ 7.30-7.36 (m, 4H) 7.21-7.26 (m, 1H) 5.68 (dt, $J=15.5,7.0 \mathrm{~Hz}, 1 \mathrm{H}) 5.59$ (ddt, $J=15.3,6.5,1.0 \mathrm{~Hz}, 1 \mathrm{H}) 5.15(\mathrm{~d}, J=7.0 \mathrm{~Hz}, 1 \mathrm{H})$ $3.61(\mathrm{t}, J=6.5 \mathrm{~Hz}, 2 \mathrm{H}) 2.07(\mathrm{q}, J=7.0 \mathrm{~Hz}, 2 \mathrm{H}) 1.83-2.00(\mathrm{~m}, 1 \mathrm{H}) 1.57$ (quint, $J=6.5$ $\mathrm{Hz}, 2 \mathrm{H})$ 1.43-1.49 (m, 2H) 0.93-0.97 (m, 9H) 0.56-0.68 (m, 6H); ${ }^{13} \mathrm{C}\left(125 \mathrm{MHz}, \mathrm{CDCl}_{3}\right)$ $\delta 144.7,134.2,130.5,128.3,127.1,126.1,75.7,62.9,32.5,32.1,25.5$. IR (film, $\mathrm{cm}^{-1}$ ) 3378.0, 2951.9, 2875.8, 1454.1, 1240.4, 1057.6. HRMS (EI) $\mathrm{m} / \mathrm{z}$ calculated for $\mathrm{C}_{19} \mathrm{H}_{32} \mathrm{O}_{2} \mathrm{Si} 320.2172$, found $320.2170\left(\mathrm{M}^{+}\right)$.

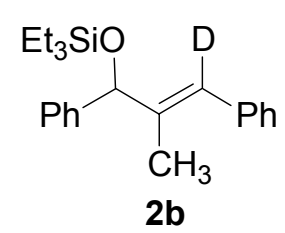

\section{Triethyl-(3-deuterio-2-methyl-1,3-diphenyl-allyloxy)-silane (2b)}

Following the general procedure, benzaldehyde $(25 \mu \mathrm{L}, 0.25 \mathrm{mmol})$, 1-phenyl-propyne (38 $\mu \mathrm{L}, 0.3 \mathrm{mmol}), \mathrm{Ni}(\mathrm{COD})_{2}(7 \mathrm{mg}, 0.025 \mathrm{mmol})$, imidazolium salt 1 (9 mg, 0.025 mmol), $\mathrm{n}-\mathrm{BuLi}(16 \mu \mathrm{L}, 0.025 \mathrm{mmol})$ and $\mathrm{Et}_{3} \mathrm{SiD}(80 \mu \mathrm{L}, 0.5 \mathrm{mmol})$ were employed to give $\mathbf{2 b}(68 \mathrm{mg}, 80 \%)$ as a colorless oil after chromatography (Hexane) ${ }^{1} \mathrm{H}$ NMR (500 $\left.\mathrm{MHz}, \mathrm{CDCl}_{3}\right) \delta 7.45(\mathrm{~d}, J=7.0 \mathrm{~Hz}, 2 \mathrm{H}), 7.32-7.37(\mathrm{~m}, 6 \mathrm{H}), 7.22-7.28(\mathrm{~m}, 2 \mathrm{H}), 5.28(\mathrm{~s}$, 1H), $1.70(\mathrm{~s}, 3 \mathrm{H}), 0.99$ (t, $J=8.0 \mathrm{~Hz}, 9 \mathrm{H}), 0.68(\mathrm{q}, J=8.0 \mathrm{~Hz}, 6 \mathrm{H}) ;{ }^{13} \mathrm{C}(125 \mathrm{MHz}$, 
$\left.\mathrm{CDCl}_{3}\right) \delta 144.6,141.0,138.0,129.2,128.3,127.1,126.6,126.4,125.4$ (t, $\left.J=23 \mathrm{~Hz}\right), 80.1$, 13.5, 7.1, 5.1; IR (film) 2954, 2911, 2874, 1597, 1493, 1065, $744 \mathrm{~cm}^{-1}$; HRMS (EI) $\mathrm{m} / \mathrm{z}$ calcd for $\mathrm{C}_{22} \mathrm{H}_{29} \mathrm{OSiD}\left[\mathrm{M}^{+}\right]=339.2129$, found 339.2125 .<smiles></smiles>

2c

\section{Tripropyl-(2-methyl-1,3-diphenyl-allyloxy)-silane (2c)}

Following the general procedure, benzaldehyde $(102 \mu \mathrm{L}, 1.0 \mathrm{mmol})$, 1-phenyl-propyne $(150 \mu \mathrm{L}, 1.2 \mathrm{mmol}), \mathrm{Ni}(\mathrm{COD})_{2}(28 \mathrm{mg}, 0.1 \mathrm{mmol})$, imidazolium salt 1 (34 mg, 0.1 mmol), n-BuLi $(63 \mu \mathrm{L}, 0.1 \mathrm{mmol})$ and $\operatorname{Pr}_{3} \operatorname{SiH}(417 \mu \mathrm{L}, 2.0 \mathrm{mmol})$ were employed to give 2c ( $298 \mathrm{mg}, 78 \%$ ) as a colorless oil after chromatography (Hexane) ${ }^{1} \mathrm{H}$ NMR (400 $\left.\mathrm{MHz}, \mathrm{CDCl}_{3}\right) \delta 7.42(\mathrm{~d}, J=8.0 \mathrm{~Hz}, 2 \mathrm{H}), 7.29-7.36(\mathrm{~m}, 6 \mathrm{H}), 7.20-7.26(\mathrm{~m}, 2 \mathrm{H}), 6.71(\mathrm{~s}$, 1H), $5.24(\mathrm{~s}, 1 \mathrm{H}), 1.66(\mathrm{~s}, 3 \mathrm{H}), 1.33-1.44(\mathrm{~m}, 6 \mathrm{H}), 0.94(\mathrm{t}, J=7.2 \mathrm{~Hz}, 9 \mathrm{H}), 0.62-0.66(\mathrm{~m}$, $6 \mathrm{H}) ;{ }^{13} \mathrm{C}$ NMR $\left(100 \mathrm{MHz}, \mathrm{CDCl}_{3}\right) \delta 143.6,141.1,138.1,129.2,128.3,128.2,127.1$, 126.6, 126.4, 125.7, 80.2, 18.7, 17.10, 17.07, 13.4; IR (film) 2953, 2923, 2866, 1600, 1491, 1450, 1059, 739, $698 \mathrm{~cm}^{-1}$; HRMS (EI) $\mathrm{m} / z$ calcd for $\mathrm{C}_{25} \mathrm{H}_{36} \mathrm{OSi}\left[\mathrm{M}^{+}\right]=380.2535$, found 380.2536 .

\section{General procedure $\mathrm{C}$ for the $\mathrm{Ni}(\mathrm{COD})_{2} /$ carbene catalyzed cyclization of ynals:}

Addition of $\mathrm{Ni}(\mathrm{COD})_{2}$ (0.1 equiv.), imidazolium salt 1 (0.1 equiv.), n-Buli (0.1 equiv.) and trialkysilane (1.1-2.0 equiv.) followed the general procedure for intermolecular couplings. Then a $0.5 \mathrm{M}$ THF solution of ynal (1.0 equiv.) was added dropwise. The mixture was stirred for $10 \mathrm{~min}$ at $45^{\circ} \mathrm{C}$, followed by quenching with sat. $\mathrm{NaHCO}_{3}$ and 
extracting $3 \mathrm{x}$ with $\mathrm{Et}_{2} \mathrm{O}$. The combined organic layers were washed with brine, dried with $\mathrm{MgSO}_{4}$, filtered, and concentrated by a rotary evaporation. The crude reaction mixture was purified by column chromatography over silica gel using hexane/ethyl acetate as the eluent.

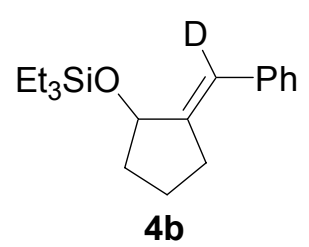

\section{2-(1-deuteriobenzylidene)-cyclopentyloxy-triethyl-silane (4b)}

Following the general procedure, 6-phenyl-hex-5-ynal (90 mg, $0.52 \mathrm{mmol}), \mathrm{Ni}(\mathrm{COD})_{2}$ (14 mg, $0.05 \mathrm{mmol})$, imidazolium salt $1(17 \mathrm{mg}, 0.05 \mathrm{mmol})$, n-BuLi $(31 \mu \mathrm{L}, 0.05$ mmol), and $\mathrm{Et}_{3} \mathrm{SiD}(91 \mu \mathrm{L}, 0.57 \mathrm{mmol})$ were employed to give $\mathbf{4 b}(95 \mathrm{mg}, 63 \%)$ as a colorless oil after chromatography (hexane/ethyl acetate: 20:1). ${ }^{1} \mathrm{H}$ NMR $(500 \mathrm{MHz}$, $\left.\mathrm{CDCl}_{3}\right) \delta$ 7.30-7.36 (m, 4H), 7.16-7.20 (m, 1H), $4.56(\mathrm{t}, J=7.0 \mathrm{~Hz}, 1 \mathrm{H}), 2.57-2.71(\mathrm{~m}$, 2H), 1.89-1.97 (m, 2H), 1.51-1.70 (m, 2H), 1.02 (t, $J=8.0 \mathrm{~Hz}, 9 \mathrm{H}), 0.69$ (q, $J=8.0 \mathrm{~Hz}, 6$ $\mathrm{H}) ;{ }^{13} \mathrm{C}$ NMR $\left(125 \mathrm{MHz}, \mathrm{CDCl}_{3}\right) \delta 147.1,138.4,128.6,128.4,126.3,77.1,35.2,28.8$, 21.7, 7.1, 5.2; IR (film) 2956, 2910, 2875, $1491 \mathrm{~cm}^{-1}$; HRMS (EI) $\mathrm{m} / z$ calcd for $\mathrm{C}_{18} \mathrm{H}_{27} \mathrm{OSiD}\left[\mathrm{M}^{+}\right]=289.1972$, found 289.1970.

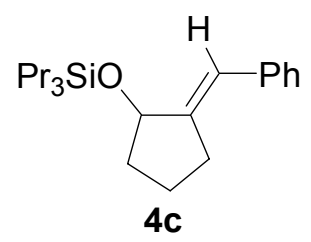

\section{(2-Benzylidene-cyclopentyloxy)-tripropyl-silane (4c)}

Following the general procedure, 6-phenyl-hex-5-ynal (150 mg, $0.87 \mathrm{mmol}), \mathrm{Ni}(\mathrm{COD})_{2}$ (25 mg, $0.09 \mathrm{mmol}$ ), imidazolium salt 1 (31 mg, $0.09 \mathrm{mmol})$, n-BuLi (56 $\mu \mathrm{L}, 0.09$ mmol), and $\operatorname{Pr}_{3} \mathrm{SiH}(378 \mu \mathrm{L}, 1.74 \mathrm{mmol})$ were employed to give $4 \mathbf{c}(224 \mathrm{mg}, 78 \%)$ as a 
colorless oil after chromatography (hexane/ethyl acetate: $20: 1) .{ }^{1} \mathrm{H}$ NMR $(500 \mathrm{MHz}$, $\left.\mathrm{CDCl}_{3}\right) \delta$ 7.30-7.36 (m, 4H), 7.17-7.20 (m, 1H), $6.45(\mathrm{q}, J=2.5 \mathrm{~Hz}, 1 \mathrm{H}), 4.54(\mathrm{~m}, 1 \mathrm{H})$, 2.56-2.70 (m, 2H), 1.88-1.95 (m, 2H), 1.60-1.70 (m, 1H), 1.52-1.57 (m, 1H), 1.40-1.48 (m, 6H), $0.99(\mathrm{t}, J=7.5 \mathrm{~Hz}, 9 \mathrm{H}), 0.67-0.70(\mathrm{~m}, 6 \mathrm{H}) ;{ }^{13} \mathrm{C} \mathrm{NMR}\left(125 \mathrm{MHz}, \mathrm{CDCl}_{3}\right)$ $\delta 147.2,138.4,128.6,128.4,126.3,122.4,77.2,35.2,28.8,21.7,18.7,17.2,17.1 ;$ IR (film) $2954,2923,2867,1061,856 \mathrm{~cm}^{-1}$; HRMS (EI) $\mathrm{m} / z$ calcd for $\mathrm{C}_{18} \mathrm{H}_{27} \mathrm{OSi}\left[\mathrm{M}^{+}\right]$ $=330.2379$, found 330.2382 .

\section{General procedure for the $\mathrm{Ni}(\mathrm{COD})_{2} / \mathrm{PBu}_{3}$ catalyzed cyclization of ynals:}

To a $0.1 \mathrm{M}$ THF solution of $\mathrm{Ni}(\mathrm{COD})_{2}$ (0.2 equiv.) was added $\mathrm{PBu}_{3}(0.4$ equiv.) at $\mathrm{rt}$. After 5 min at rt., the solution was cooled to $0^{\circ} \mathrm{C}$ and silane (2.0 equiv.) was added dropwise. The mixture was heated at $45^{\circ} \mathrm{C}$ and a $0.5 \mathrm{M}$ THF solution of ynal (1.0 equiv.) was then added dropwise. The temperature was maintained at $45^{\circ} \mathrm{C}$ for 20 hours. The reaction mixture was quenched with sat. $\mathrm{NaHCO}_{3}$ at $\mathrm{rt}$. and was extracted $3 \mathrm{x}$ with $\mathrm{Et}_{2} \mathrm{O}$. The combined organic layers were washed with brine, dried with $\mathrm{MgSO}_{4}$, filtered, and concentrated using a rotary evaporator. The crude reaction mixture subjected to column chromatography over silica gel using hexane/ethyl acetate as the eluent.

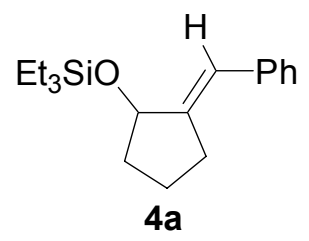

\section{(2-Benzylidene-cyclopentyloxy)-triethyl-silane (4a)}

Following the general procedure, 6-phenyl-hex-5-ynal ( $85 \mathrm{mg}, 0.49 \mathrm{mmol}), \mathrm{Ni}(\mathrm{COD})_{2}$ (28 mg, $0.1 \mathrm{mmol}), \mathrm{PBu}_{3}(50 \mu \mathrm{L}, 0.2 \mathrm{mmol})$, and $\mathrm{Et}_{3} \mathrm{SiH}(160 \mu \mathrm{L}, 1.0 \mathrm{mmol})$ were employed to give 4a (96 mg, 68\%) as a colorless oil after chromatography (hexane/ethyl 
acetate: $20: 1)^{1} \mathrm{H}$ NMR $\left(500 \mathrm{MHz}, \mathrm{CDCl}_{3}\right) \delta$ 7.31-7.36 (m, 4H), 7.17-7.20 (m, 1H), 6.48 $(\mathrm{m}, 1 \mathrm{H}), 4.56(\mathrm{~m}, 1 \mathrm{H}), 2.57-2.71(\mathrm{~m}, 2 \mathrm{H}), 1.89-1.97(\mathrm{~m}, 2 \mathrm{H}), 1.52-1.70(\mathrm{~m}, 2 \mathrm{H}), 1.02(\mathrm{t}$, $J=8.0 \mathrm{~Hz}, 9 \mathrm{H}), 0.69(\mathrm{q}, J=8.0 \mathrm{~Hz}, 6 \mathrm{H}) ;{ }^{13} \mathrm{C} \mathrm{NMR}\left(125 \mathrm{MHz}, \mathrm{CDCl}_{3}\right) \delta 147.2,138.4$, 128.6, 128.4, 126.3, 122.4, 77.1, 35.2, 28.8, 21.7, 7.1, 5.2; IR (film) 2955, 2910, 2875, $1598 \mathrm{~cm}^{-1}$; HRMS (EI) $\mathrm{m} / z$ calcd for $\mathrm{C}_{18} \mathrm{H}_{27} \mathrm{OSiD}\left[\mathrm{M}^{+}\right]=288.1909$, found 288.1909 .

\section{Crossover Experiment:}

Determination of isotopic distribution in products of catalytic crossover experiments Representative example: Table 3 with $\mathrm{PBu}_{3}$ :

Pure samples of products derived from $\mathrm{Et}_{3} \mathrm{SiH}$ (MW 288), $\mathrm{Et}_{3} \mathrm{SiD}$ (MW 289), and $\mathrm{Pr}_{3} \mathrm{SiH}$ (MW 330) were independently prepared, and GCMS analysis was performed. Based on similarity of the molecular ion regions of the $\mathrm{Et}_{3} \mathrm{SiH}$ and $\mathrm{Et}_{3} \mathrm{SiD}$-derived products, the molecular ion region of the $\mathrm{Pr}_{3} \mathrm{SiD}$-derived product was assumed to appear as the molecular ion region of the $\mathrm{Pr}_{3} \mathrm{SiH}$-derived product, shifted by one mass unit. Relative peak heights in the molecular ion region of the spectra of each pure compound were normalized, with a value of 1 assigned to the base peak.

In the crude product of an experiment that employed 1 equiv. each of $\mathrm{Et}_{3} \mathrm{SiD}$ and $\mathrm{Pr}_{3} \mathrm{SiH}$, the ratio of $\mathrm{Et}_{3} \mathrm{Si}$ products to $\mathrm{Pr}_{3} \mathrm{Si}$ products was determined by $\mathrm{GC}$ and was verified by NMR integration. From the crude GCMS, the relative intensity of the 289 and 290 peaks were normalized, with a value of 1 assigned to the base peak. The ratio of the $\mathrm{Et}_{3} \mathrm{Si}-(\mathrm{H})$ product to $\mathrm{Et}_{3} \mathrm{Si}$-(D) product was determined as follows: 
intensity of 288 peak in crossover experiment $=$

intensity of 289 peak in crossover experiment

$[\mathrm{X}] \times$ [rel height of 288 peak in pure $\mathrm{Et}_{3} \mathrm{Si}-(\mathrm{H})$ product $]+[\mathrm{Y}] \times$ [rel height of 288 peak in pure $\mathrm{Et}_{3} \mathrm{Si}-(\mathrm{D})$ product $]$

$[\mathrm{X}] \times\left[\right.$ rel height of 289 peak in pure $\mathrm{Et}_{3} \mathrm{Si}-(\mathrm{H})$ product $]+[\mathrm{Y}] \times$ [rel height of 289 peak in pure $\mathrm{Et}_{3} \mathrm{Si}-(\mathrm{D})$ product $]$

$\mathrm{X}=1 / 100 \times$ relative $\%$ of $\mathrm{Et}_{3} \mathrm{Si}-(\mathrm{H})$ product

$\mathrm{Y}=1 / 100 \times$ relative $\%$ of $\mathrm{Et}_{3} \mathrm{Si}-(\mathrm{D})$ product $=1-\mathrm{X}$

In the above equation, after substitution of $[1-\mathrm{X}]$ for $[\mathrm{Y}]$, the experimental values were inserted and the equation was solved for $[\mathrm{X}]$. The ratio of the $\mathrm{Pr}_{3} \mathrm{Si}-(\mathrm{H})$ product to $\mathrm{Pr}_{3} \mathrm{Si}_{-}$ (D) product was determined in a similar fashion. Merging the $\mathrm{GC}$ ratios of $\mathrm{Et}_{3} \mathrm{Si}$ products to $\mathrm{Pr}_{3} \mathrm{Si}$ products with the data calculated from the above equation, an overall ratio of the four possible products may be obtained. 


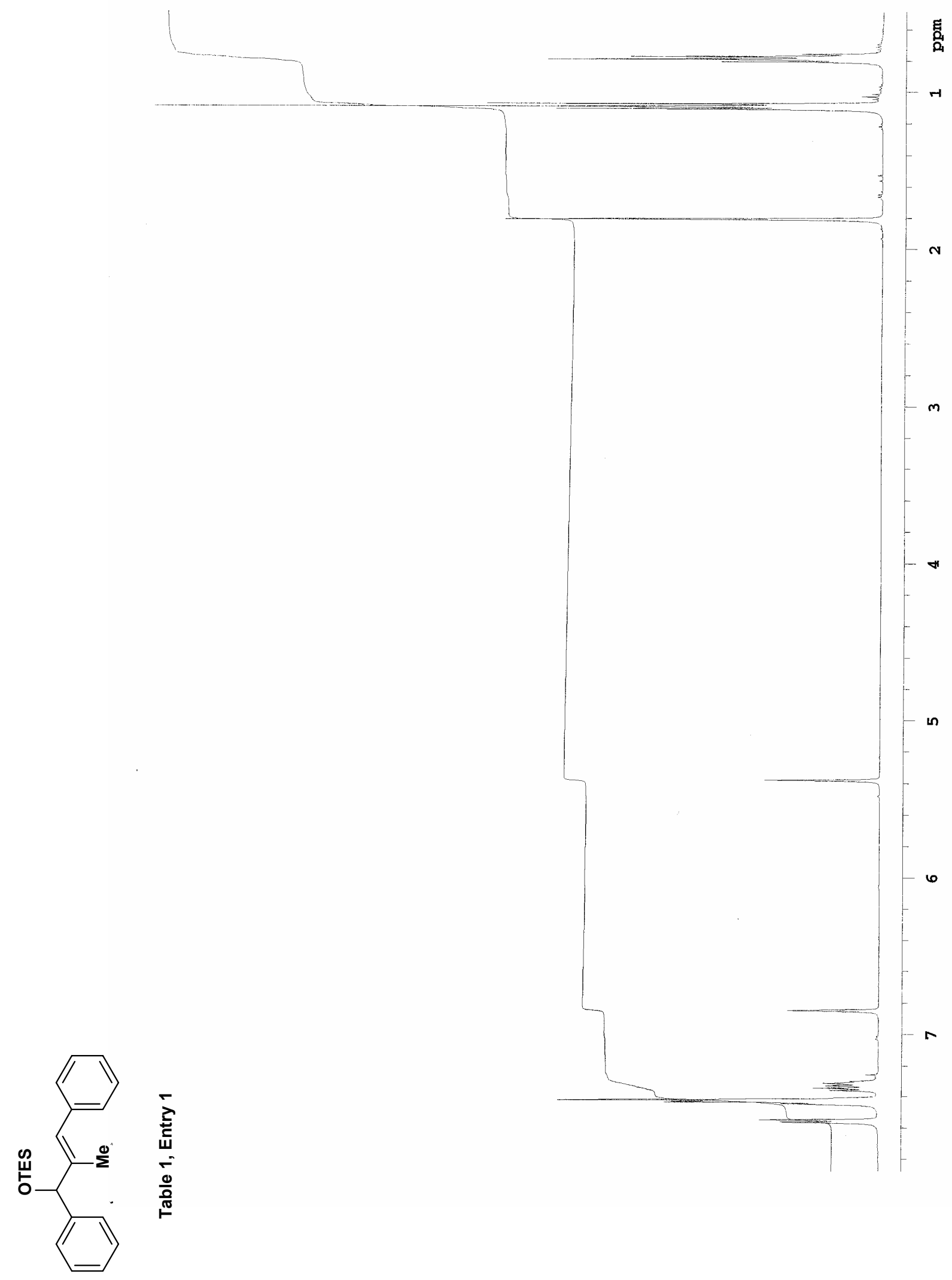




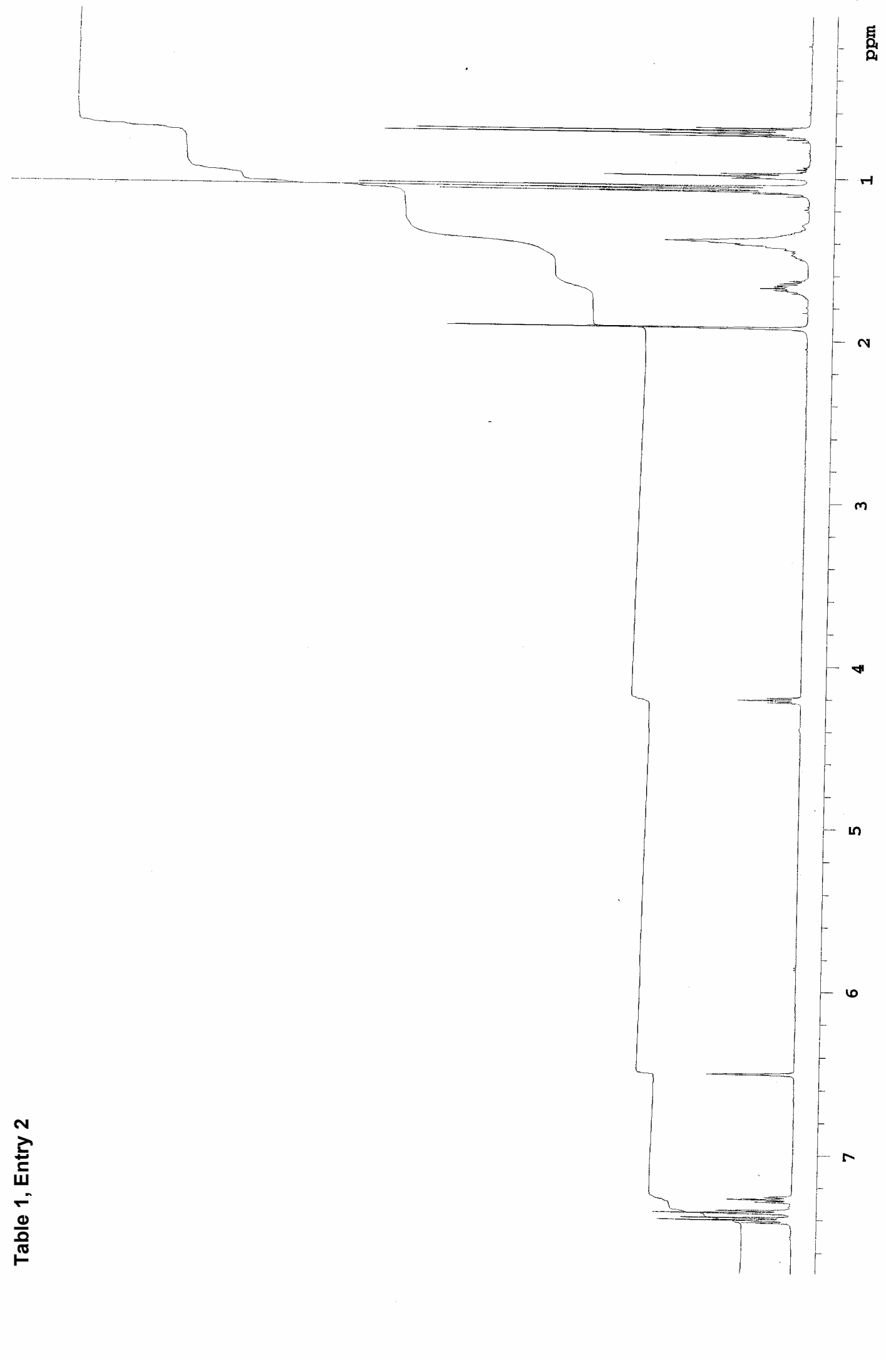




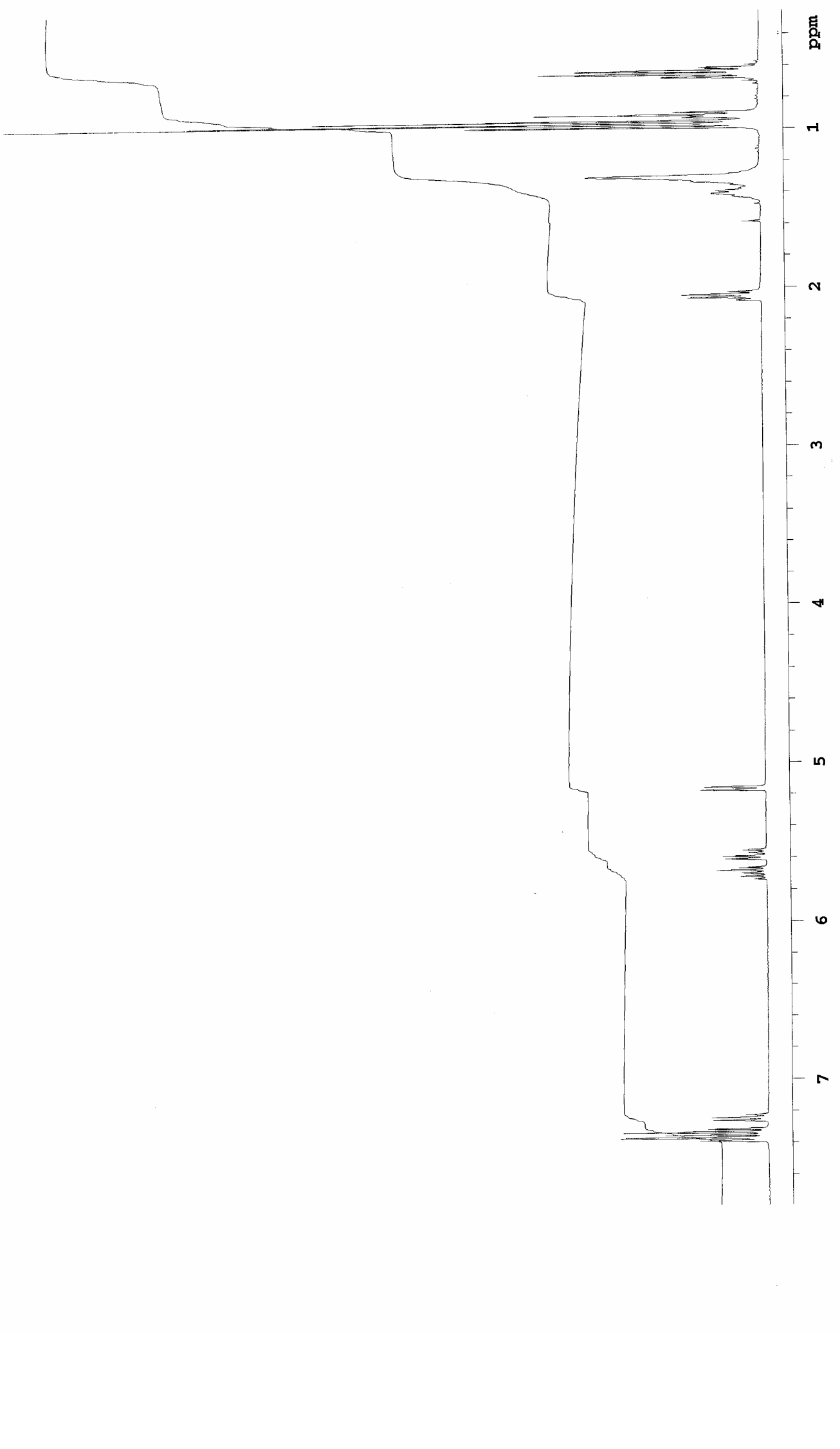




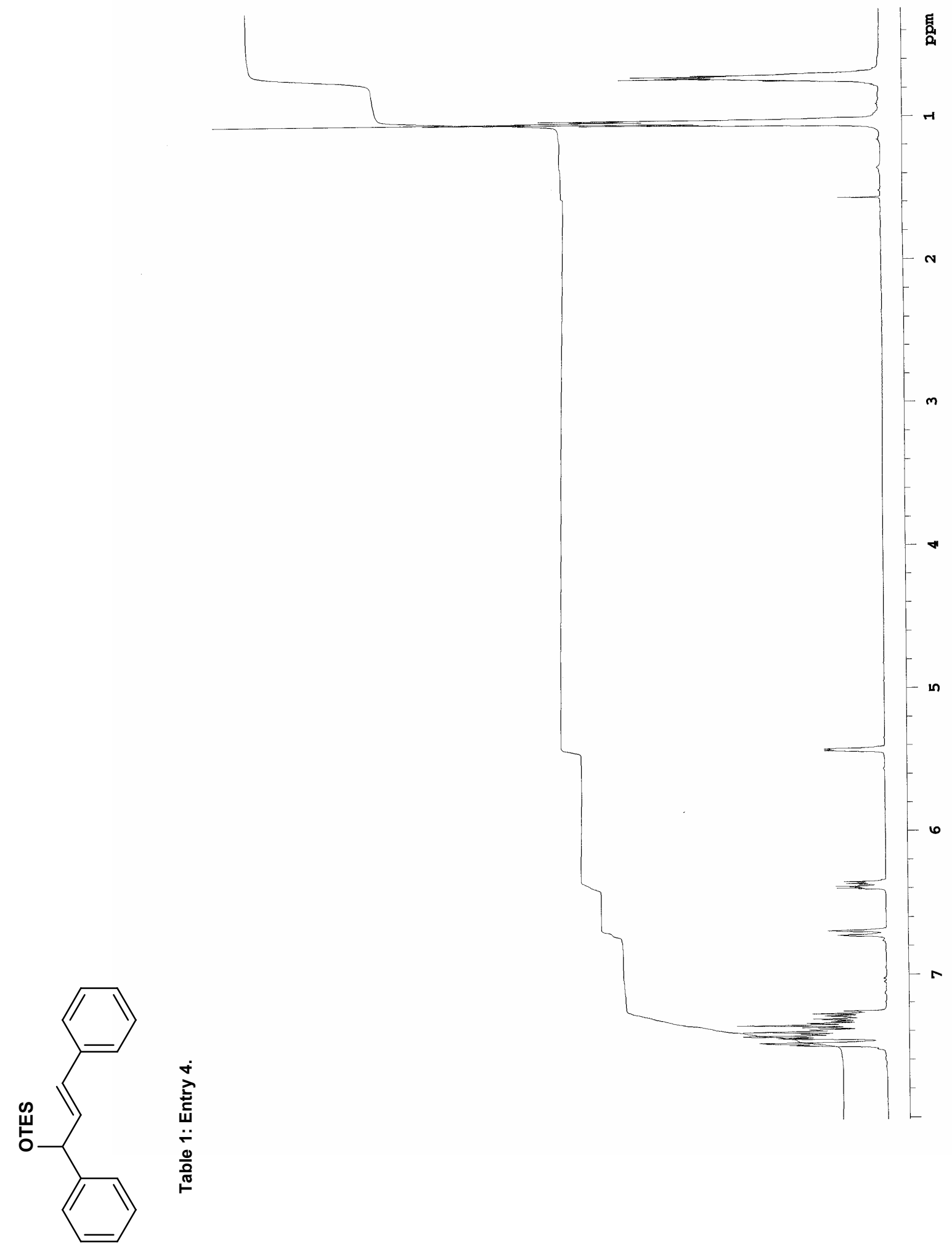




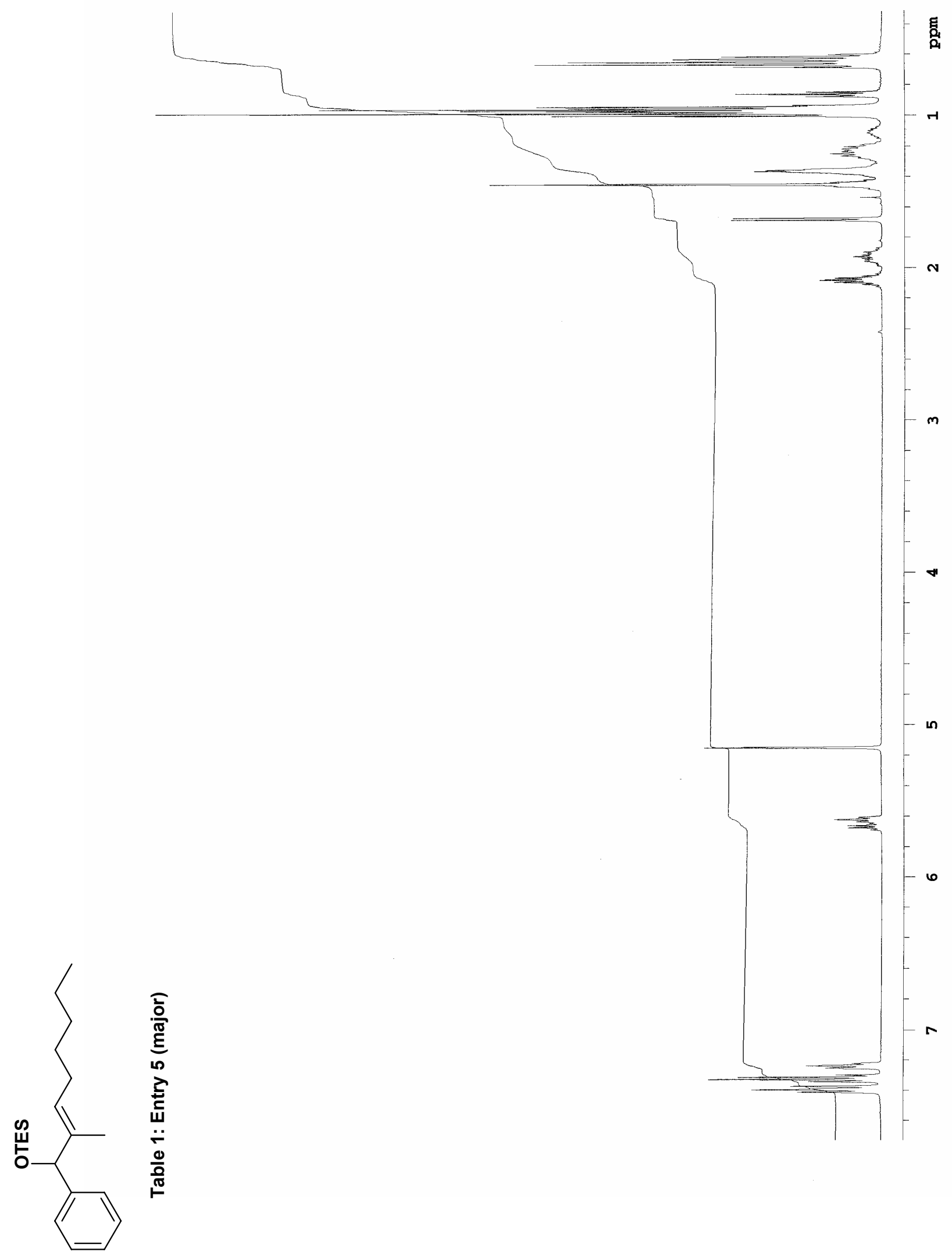




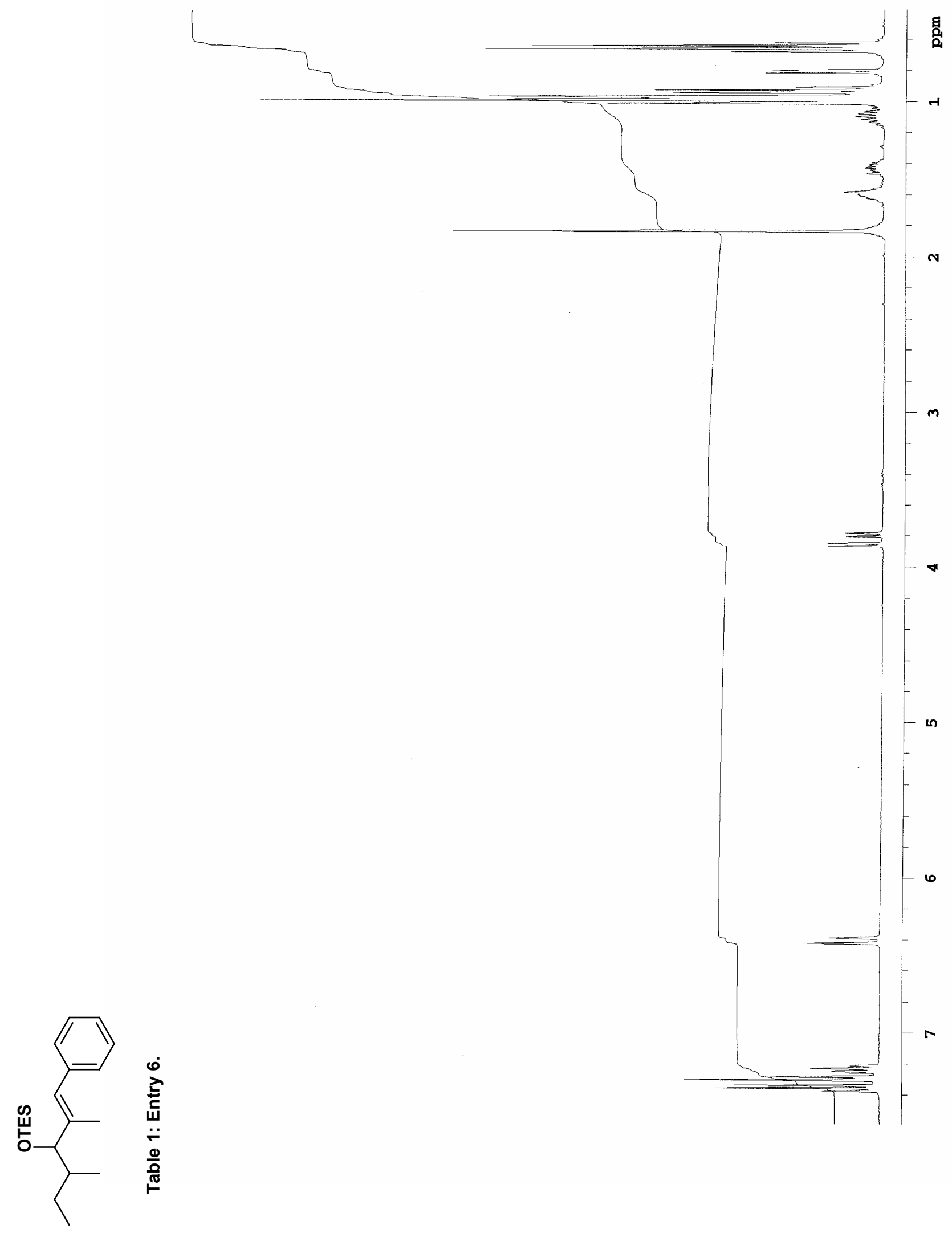




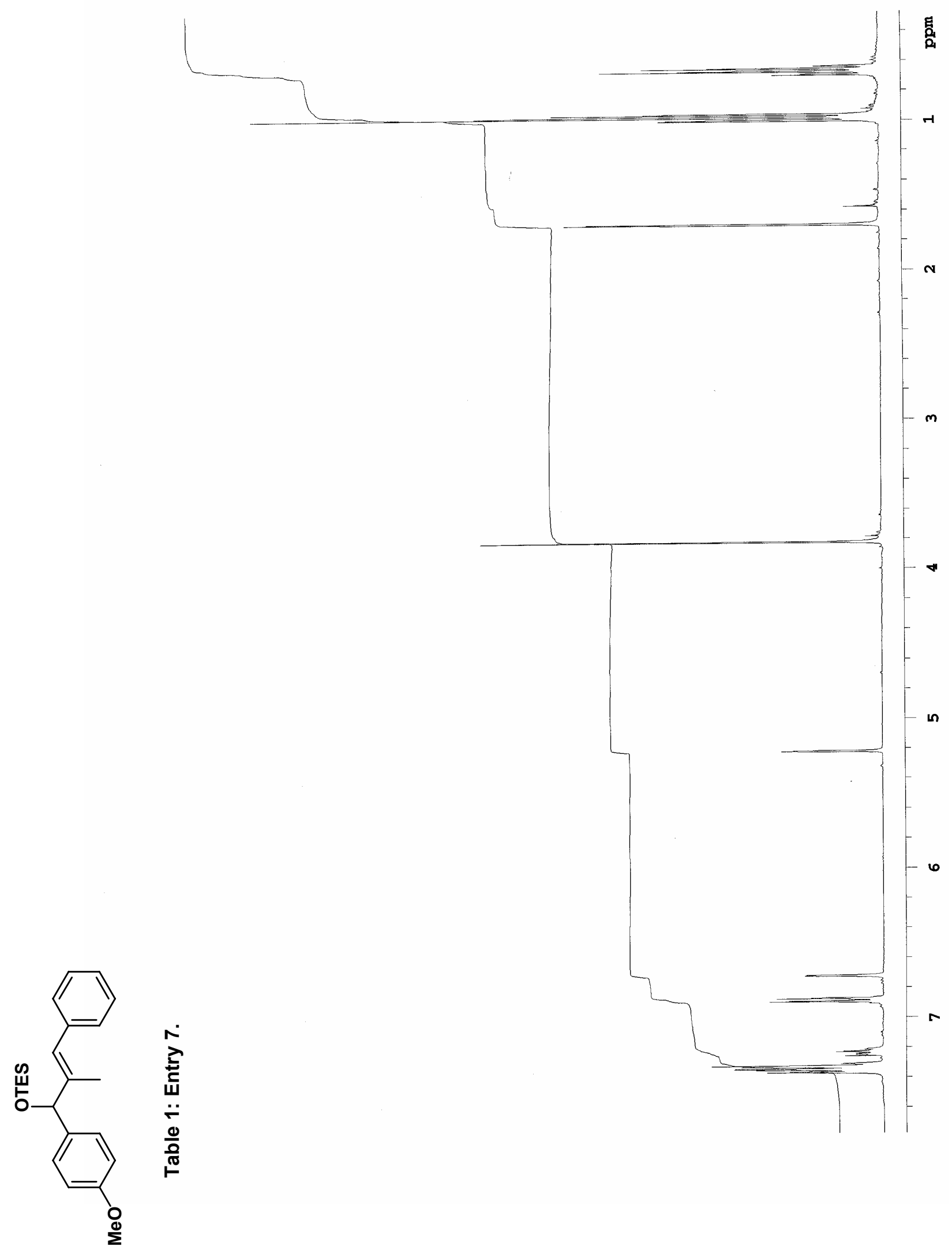




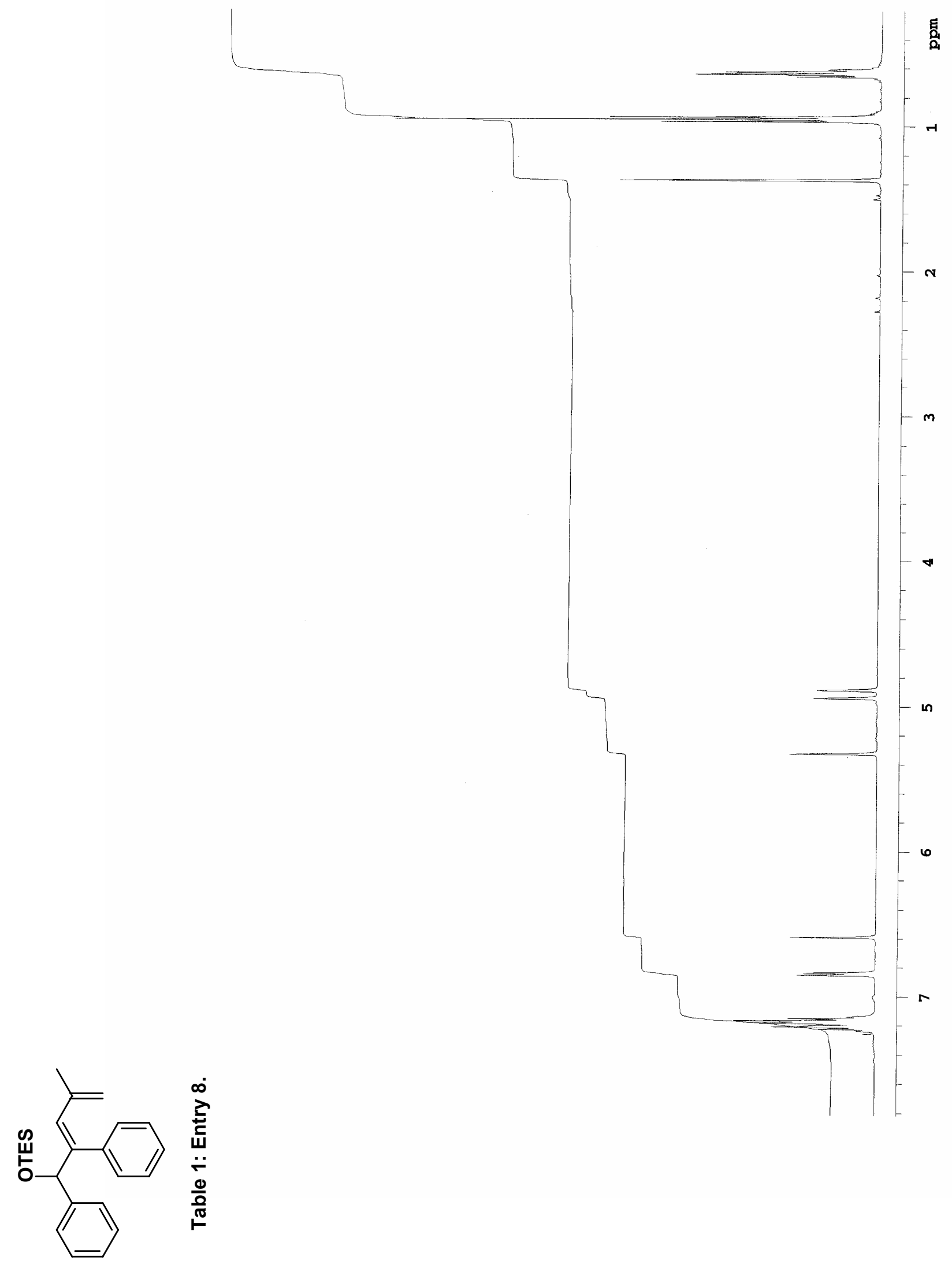




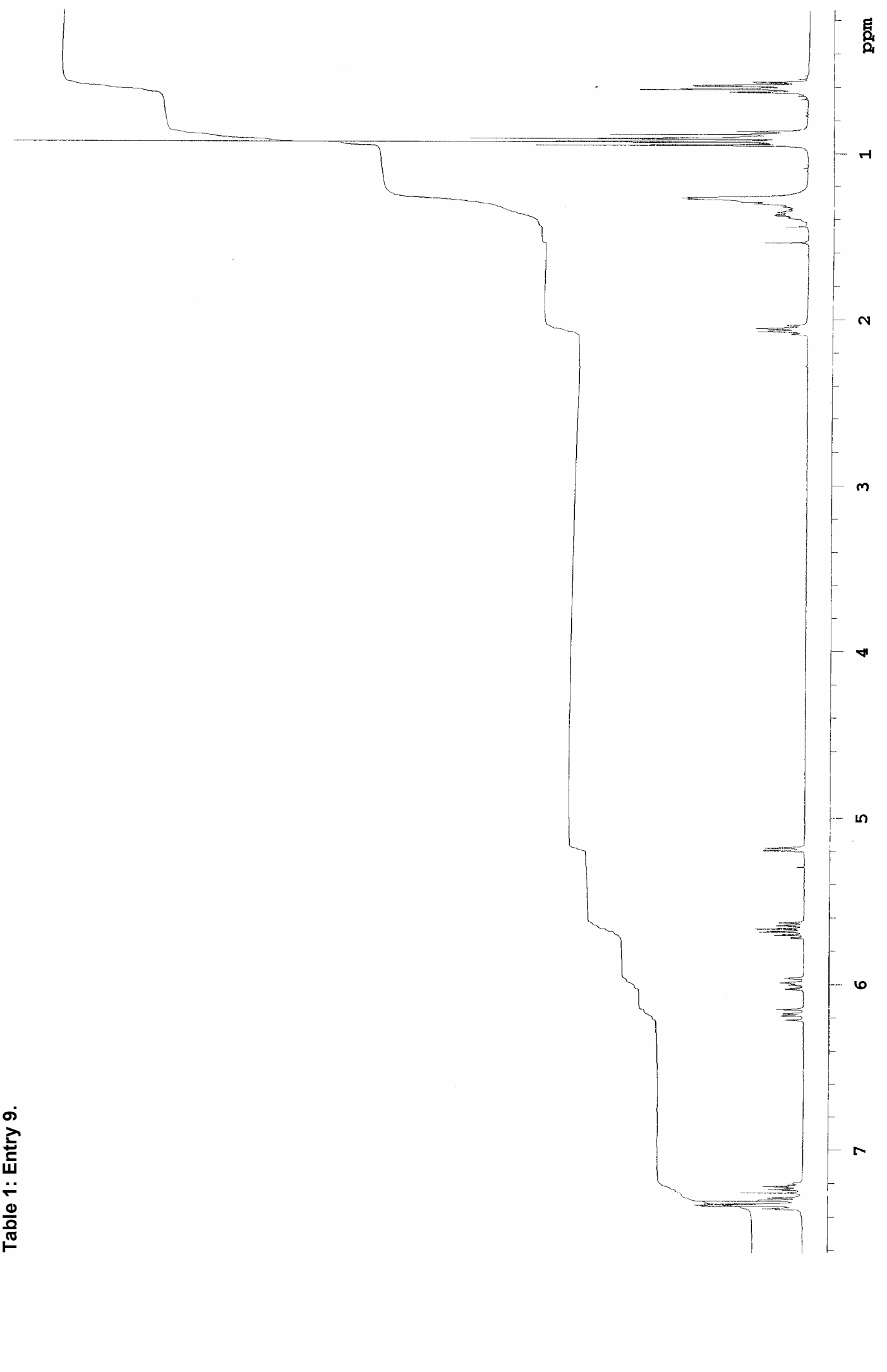




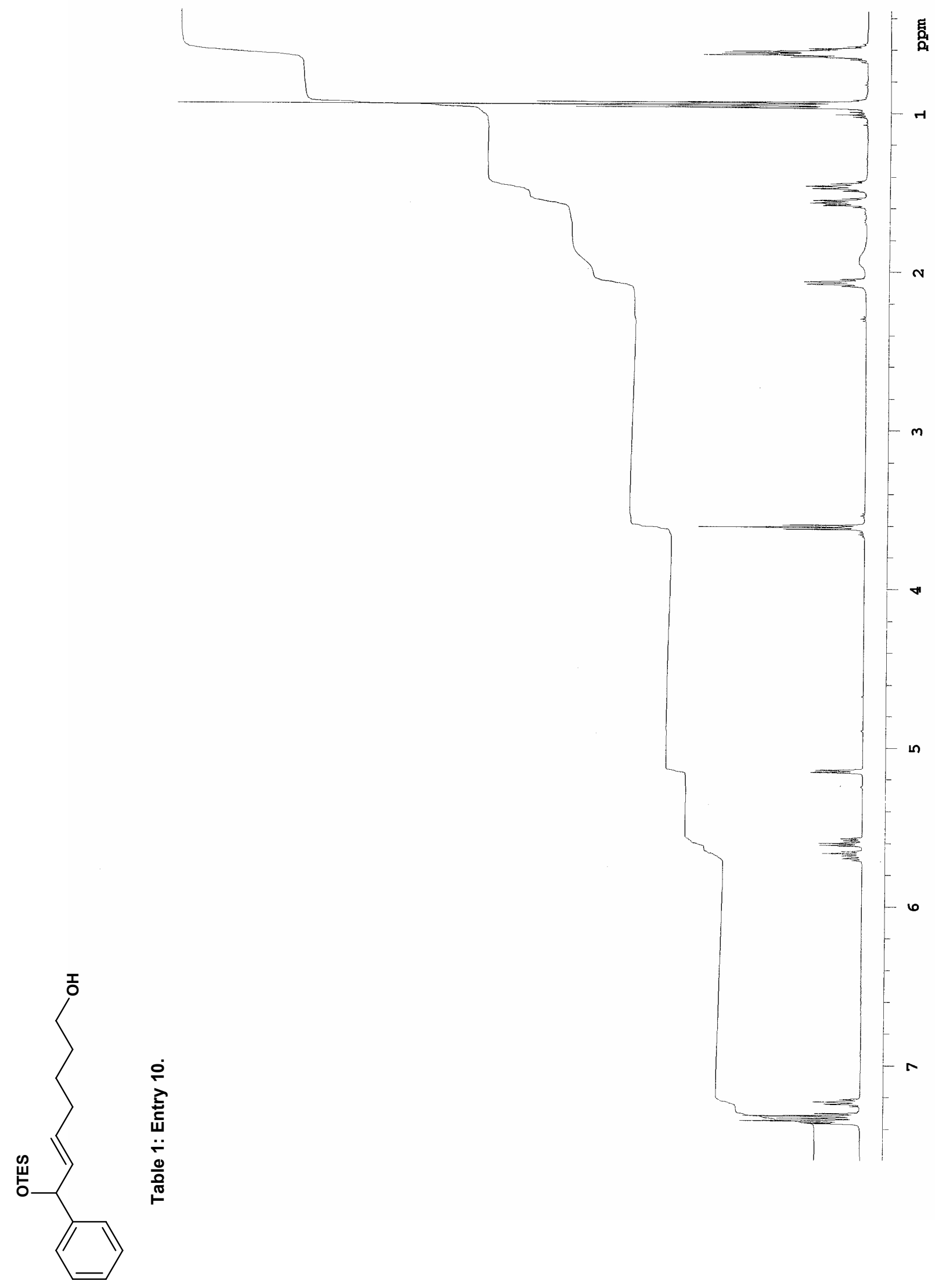



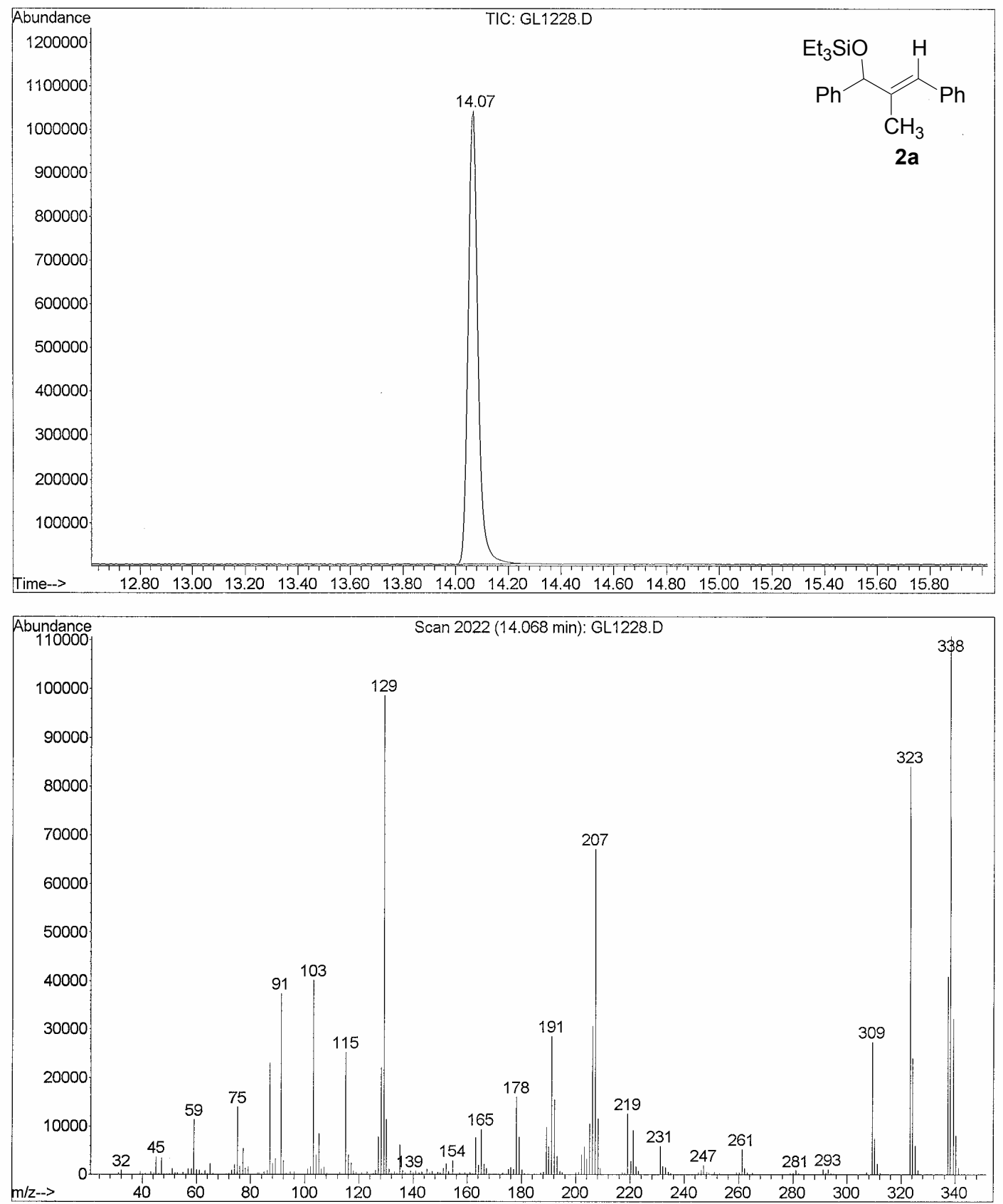

\begin{tabular}{rr}
\hline $\mathrm{M} / \mathrm{z}$ & Abundance \\
\hline 337 & 40888 \\
338 & 110688 \\
339 & 32144 \\
340 & 8132 \\
341 & 1343 \\
\hline
\end{tabular}




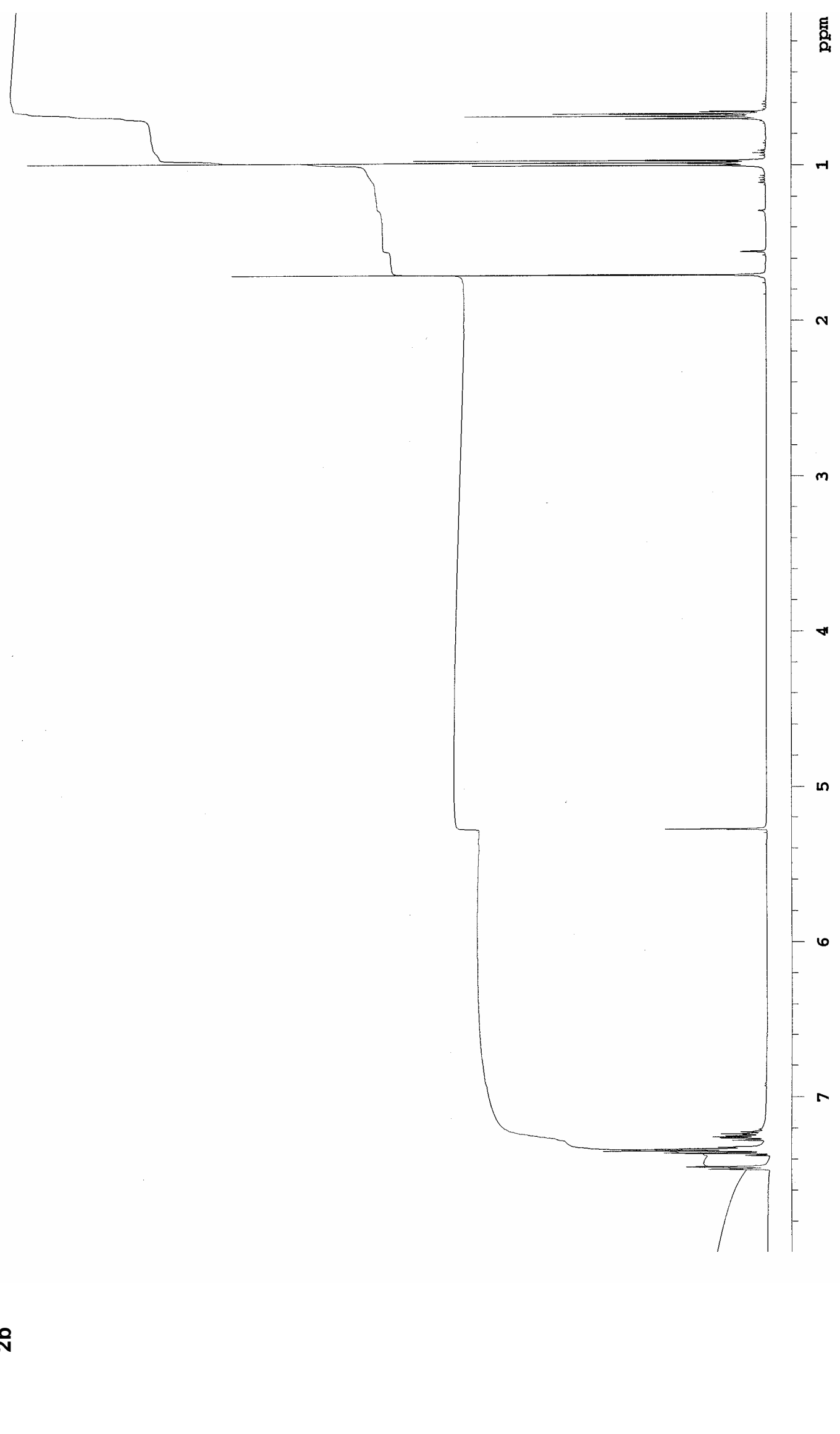



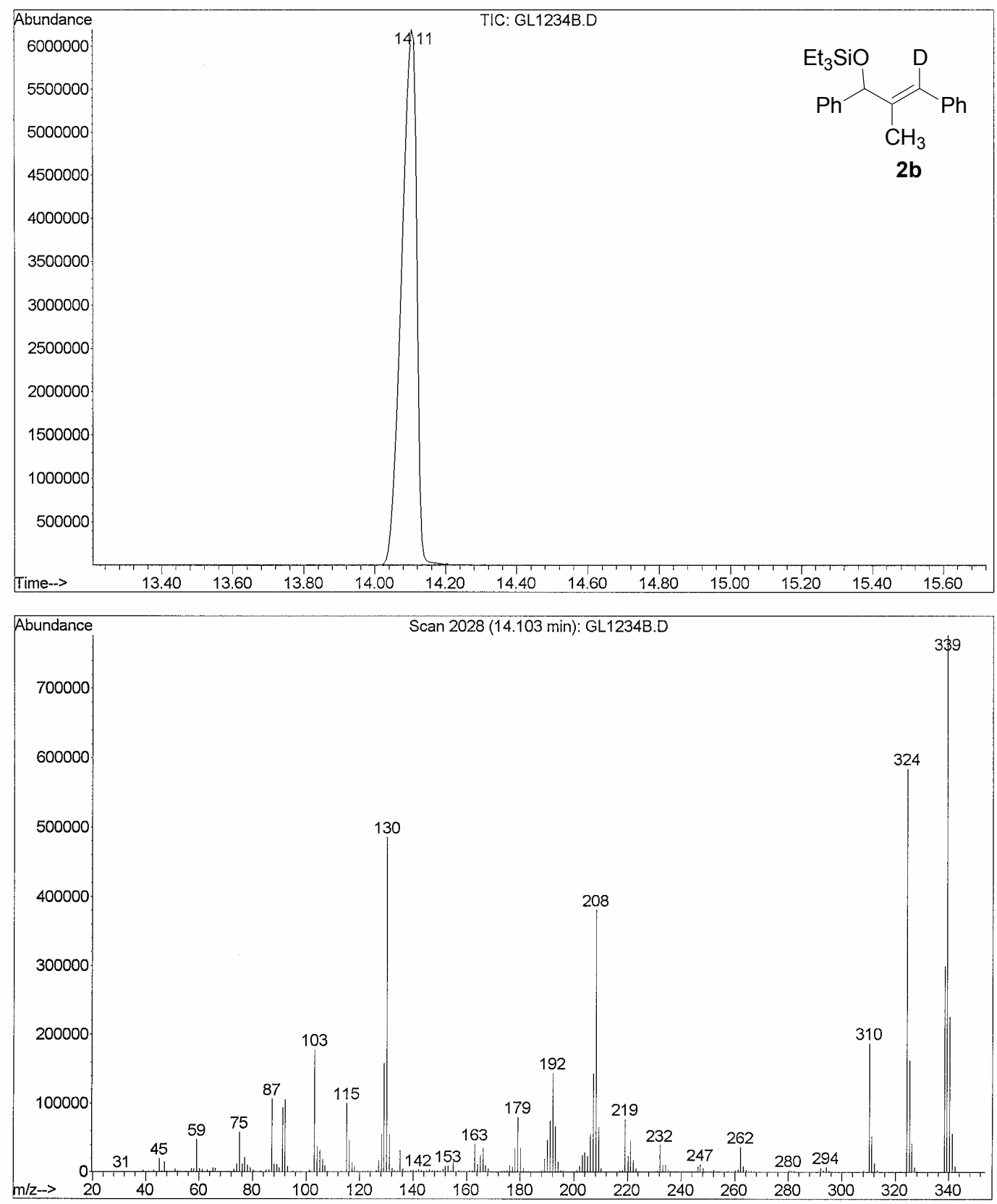

\begin{tabular}{rr}
\hline $\mathrm{M} / \mathrm{z}$ & Abundance \\
\hline 338 & 299456 \\
339 & 776576 \\
340 & 225728 \\
341 & 56808 \\
342 & 8694 \\
\hline
\end{tabular}




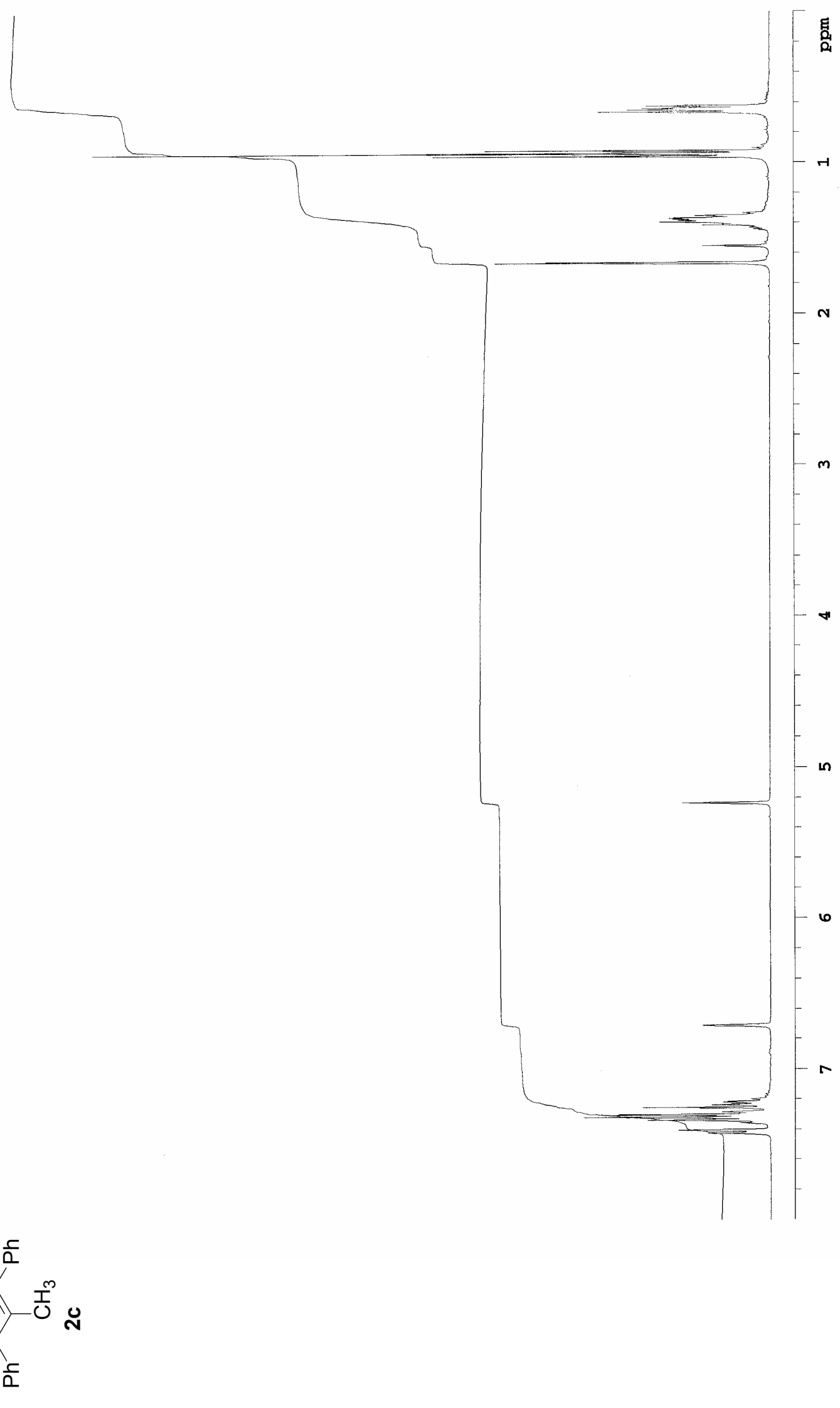



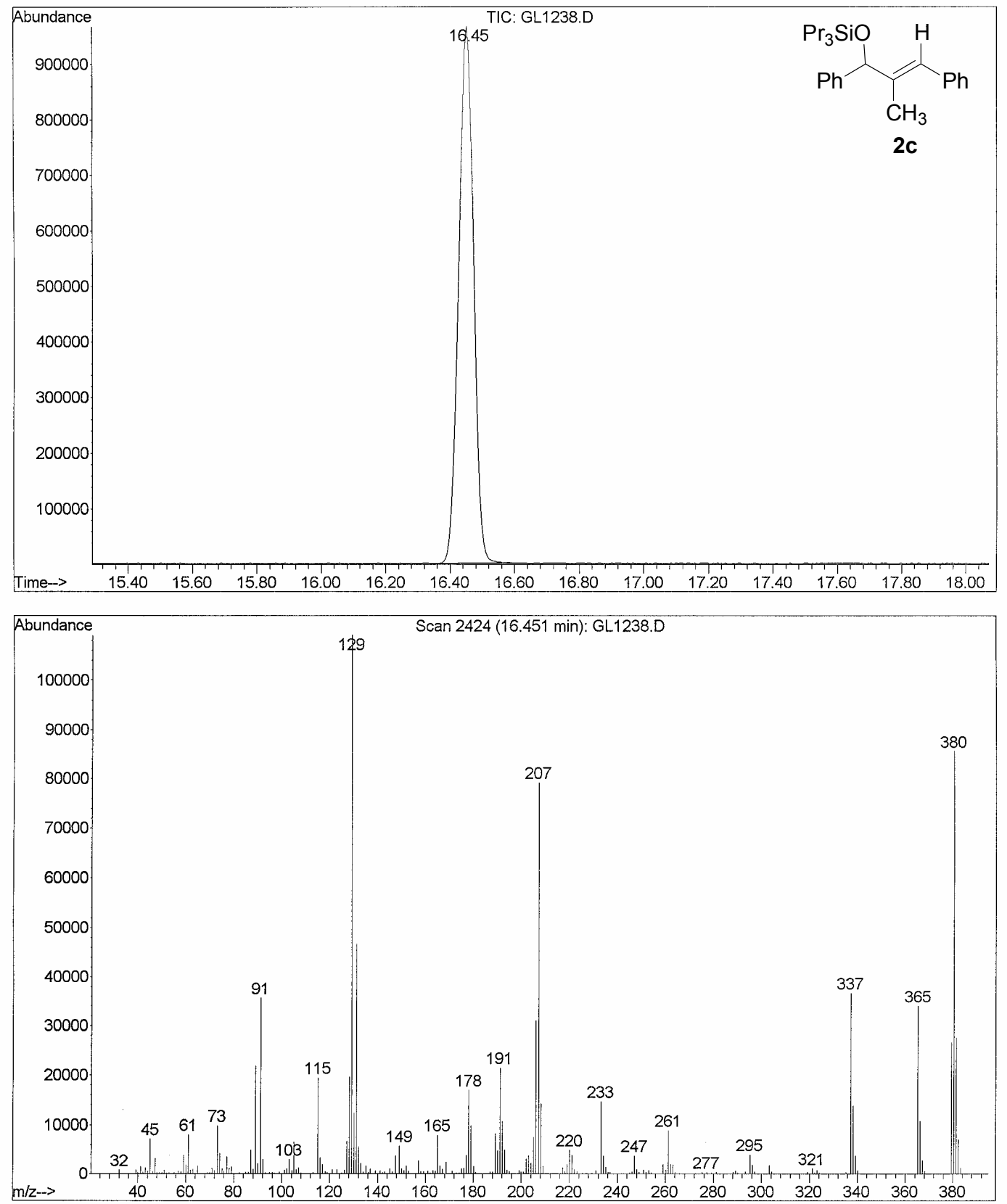

\begin{tabular}{rr}
\hline $\mathrm{M} / \mathrm{z}$ & Abundance \\
\hline 379 & 26720 \\
380 & 85624 \\
381 & 27528 \\
382 & 6985 \\
383 & 1137 \\
\hline
\end{tabular}


Crossover experiment of $\mathrm{Ni}(\mathrm{COD})_{2} /$ carbene catalyzed coupling of aldehyde and

alkyne:

$$
\text { ris }
$$

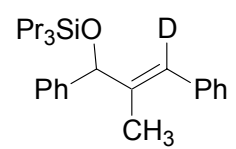

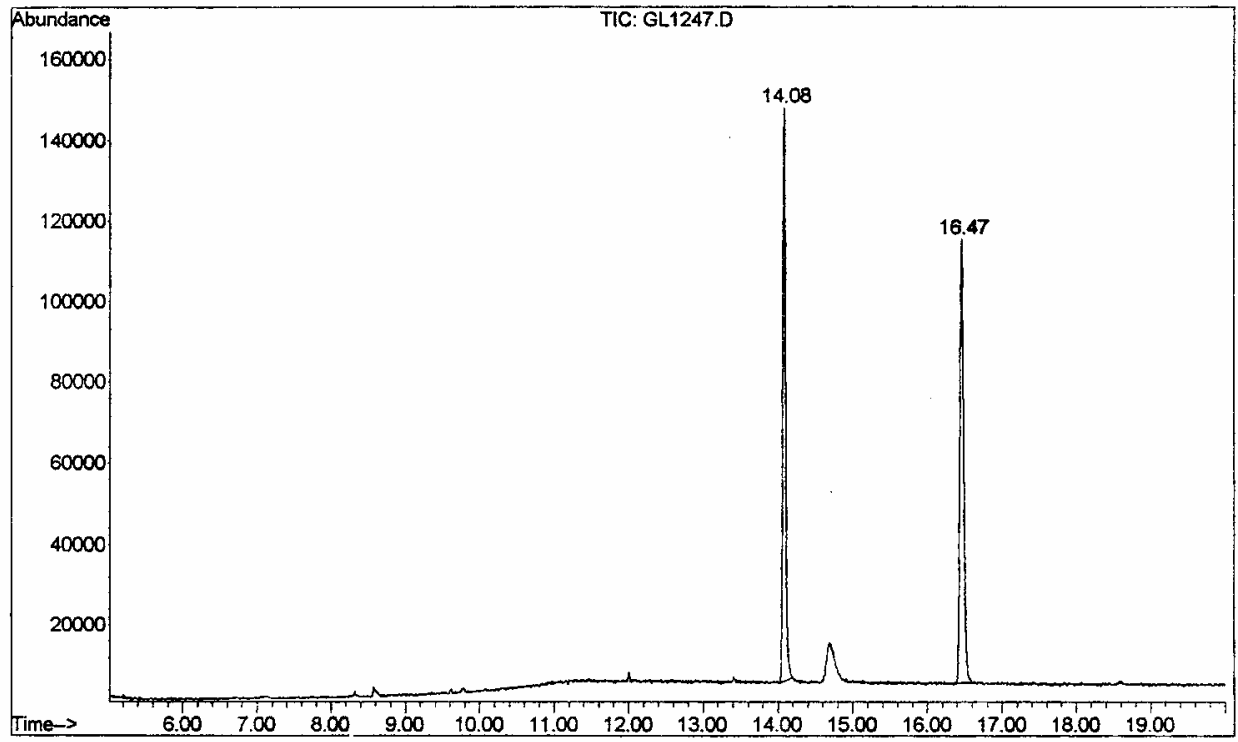




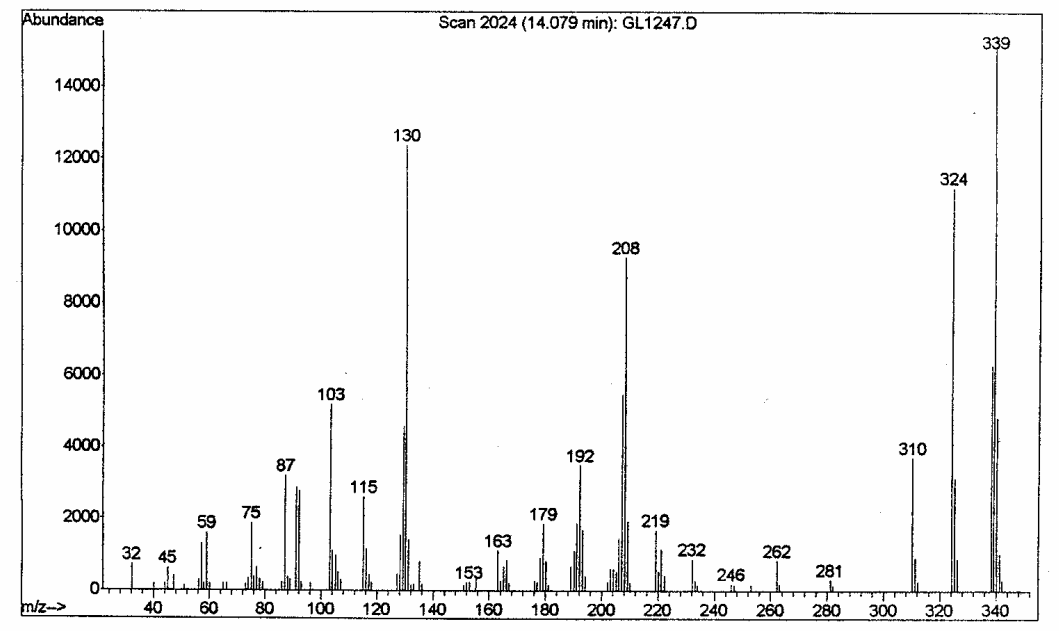

\begin{tabular}{rr}
\hline $\mathrm{M} / \mathrm{z}$ & Abundance \\
\hline 338.30 & 6289.0 \\
339.20 & 15526.0 \\
340.30 & 4844.0 \\
341.20 & 1035.0 \\
342.20 & 297.0 \\
\hline
\end{tabular}
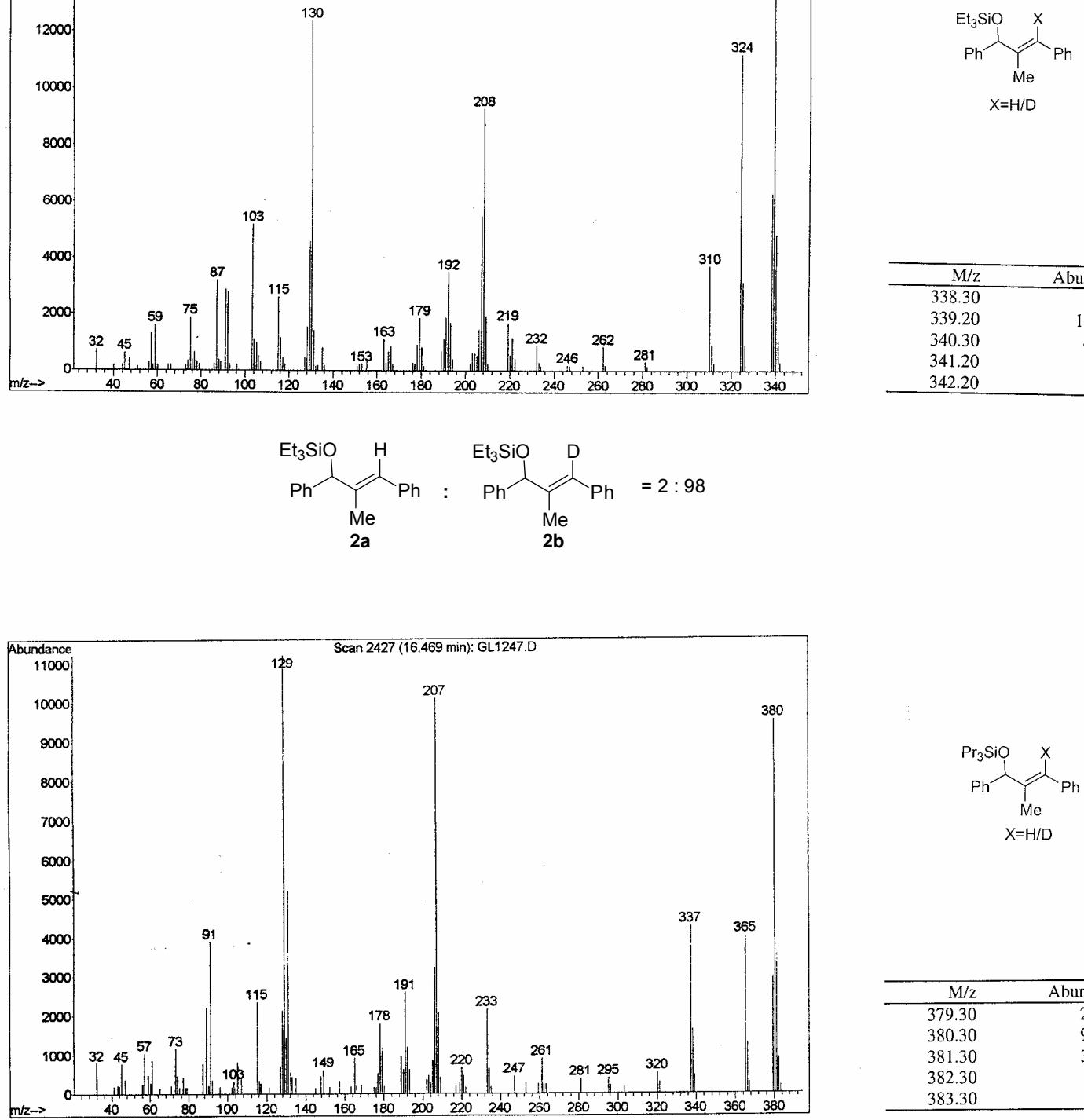

342.20

297.0

\begin{tabular}{rr}
\hline $\mathrm{M} / \mathrm{z}$ & Abundance \\
\hline 379.30 & 2970.0 \\
380.30 & 9529.0 \\
381.30 & 3568.0 \\
382.30 & 913.0 \\
383.30 & 210.0 \\
\hline
\end{tabular}

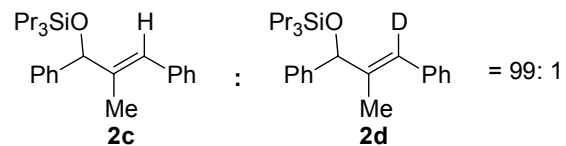


S33
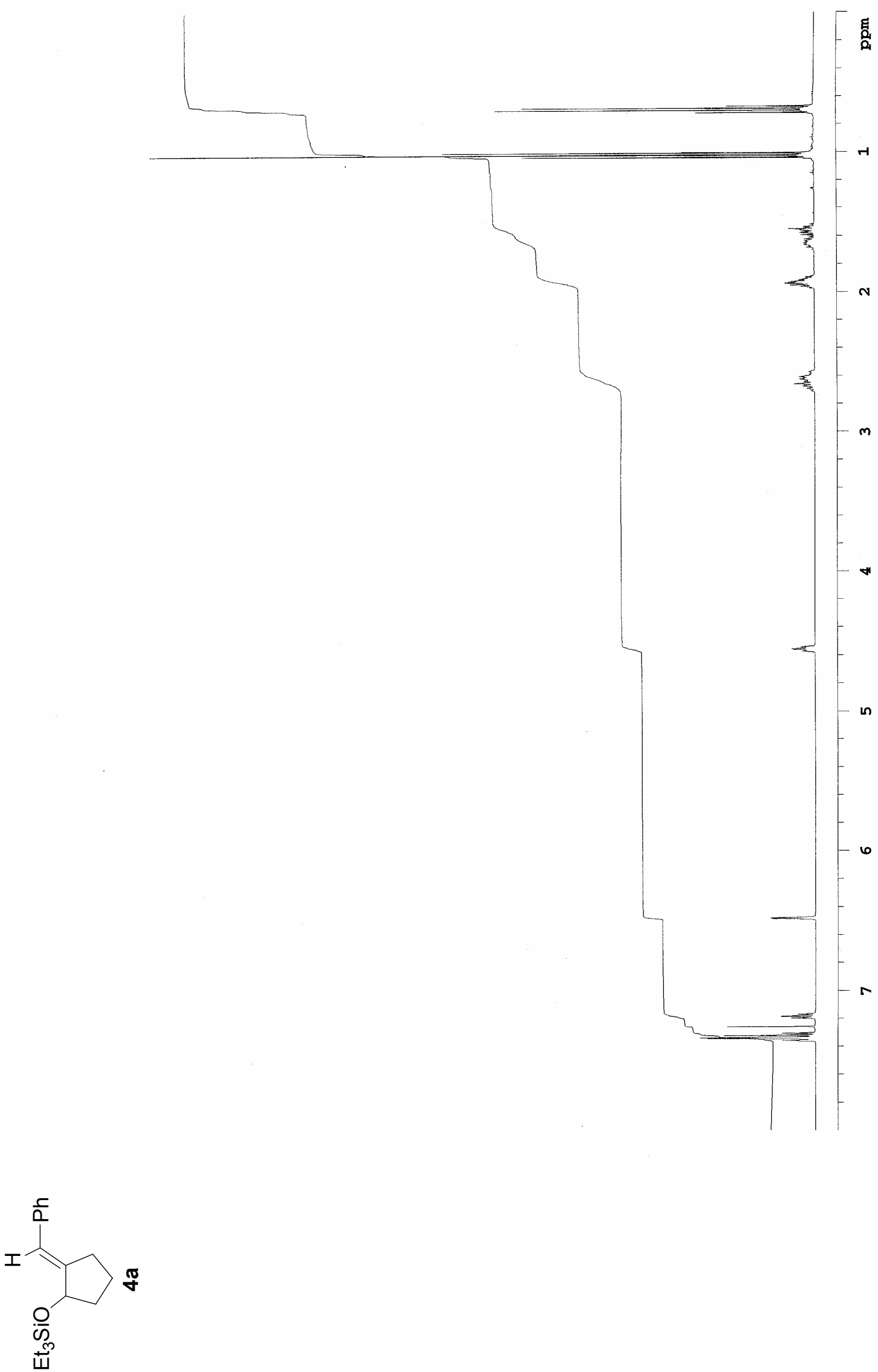

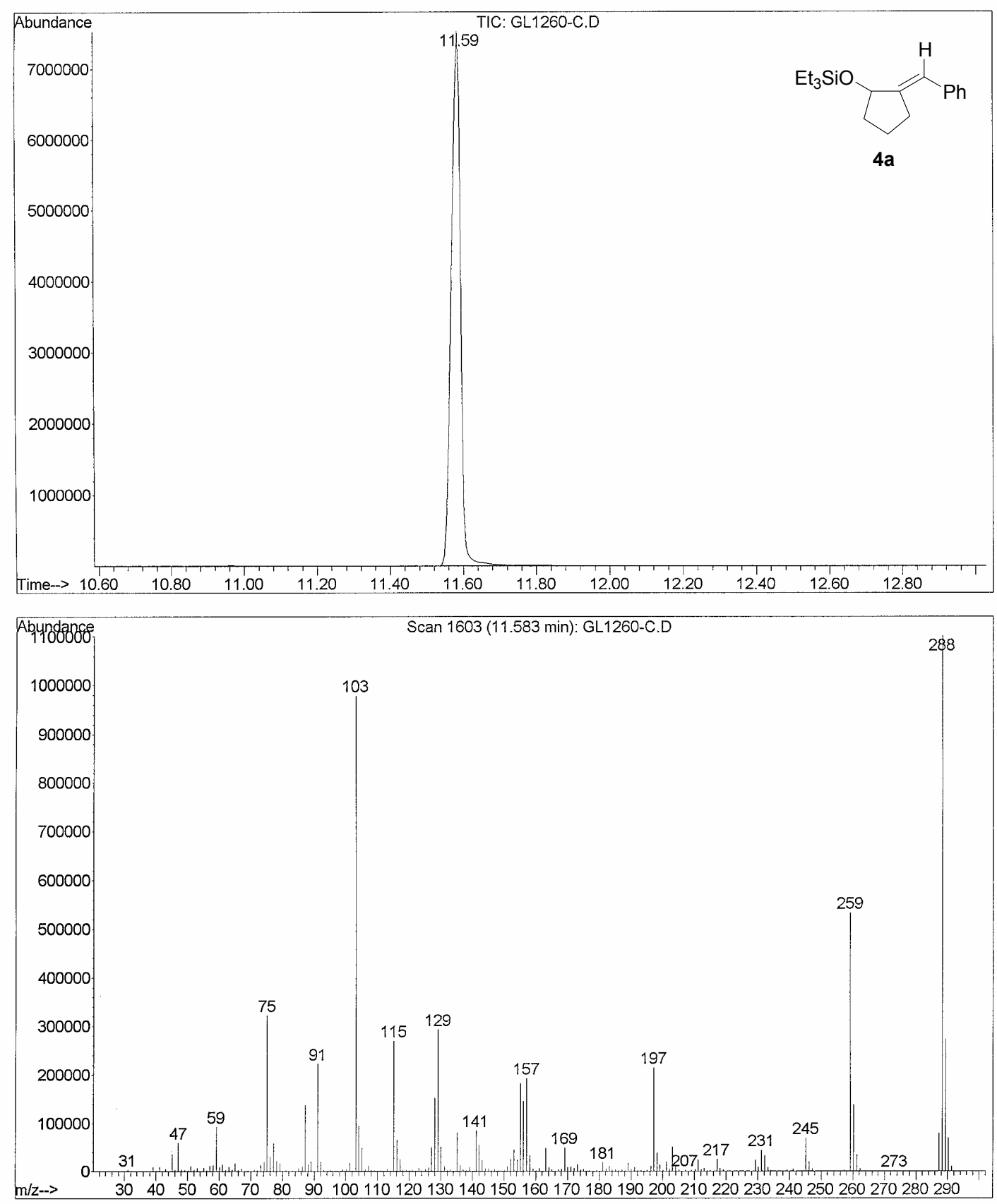

\begin{tabular}{rr}
\hline $\mathrm{M} / \mathrm{z}$ & Abundance \\
\hline 287 & 79856 \\
288 & 1100800 \\
289 & 273408 \\
290 & 70160 \\
291 & 10276 \\
292 & 1034 \\
\hline
\end{tabular}


S35

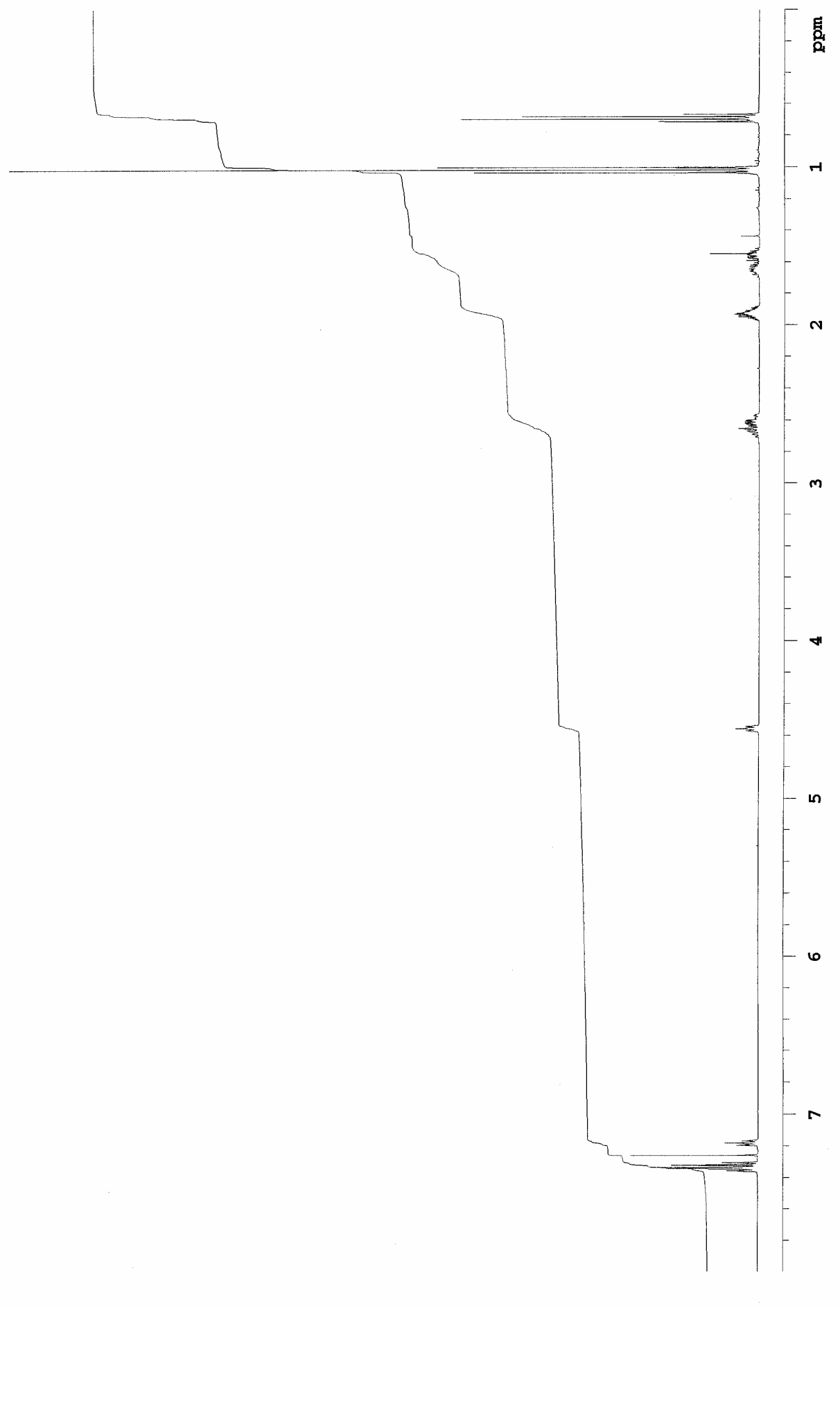



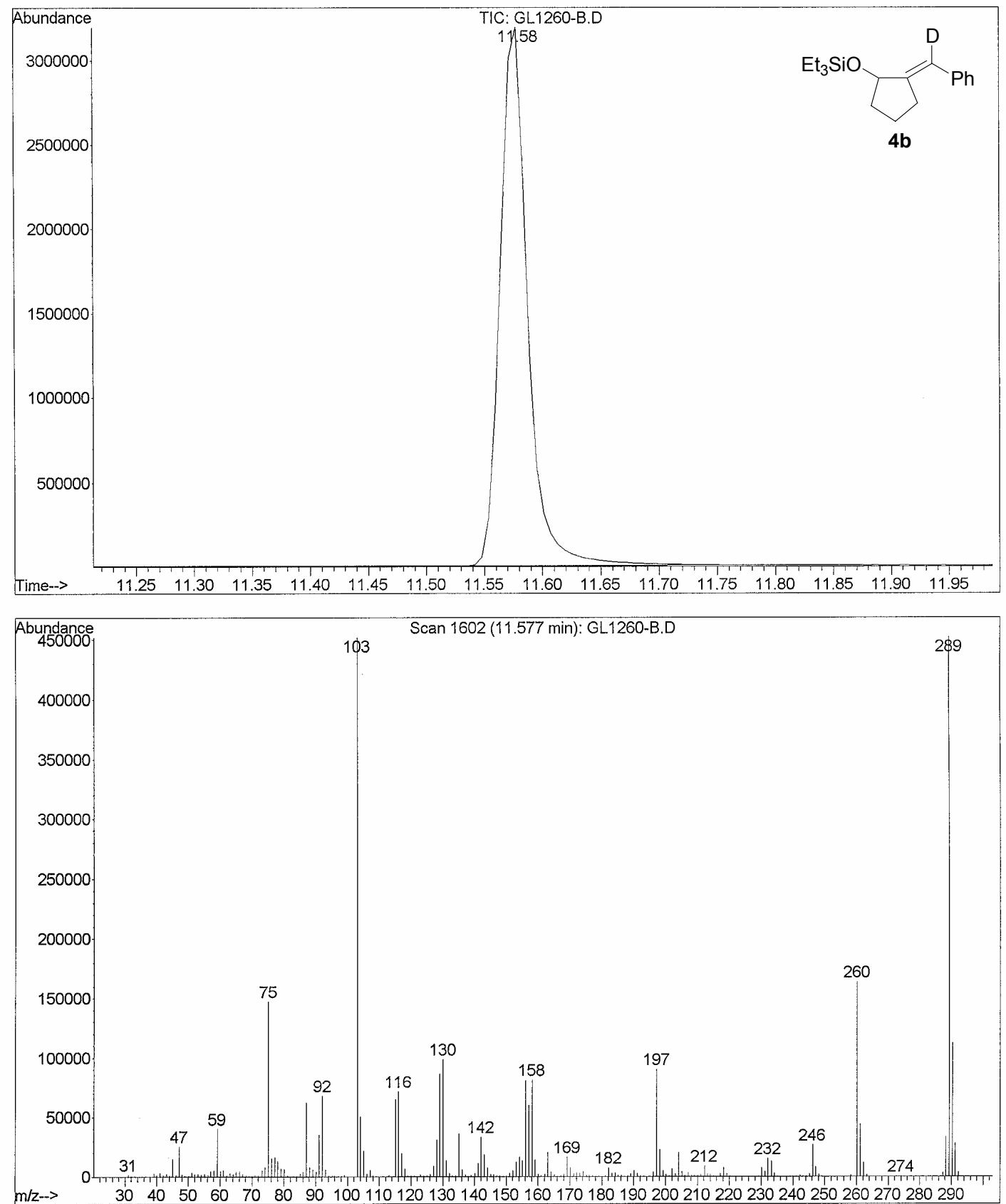

\begin{tabular}{cr}
\hline $\mathrm{M} / \mathrm{z}$ & Abundance \\
\hline 287 & 3575 \\
288 & 33784 \\
289 & 452032 \\
290 & 112624 \\
291 & 28336 \\
292 & 3828 \\
\hline
\end{tabular}



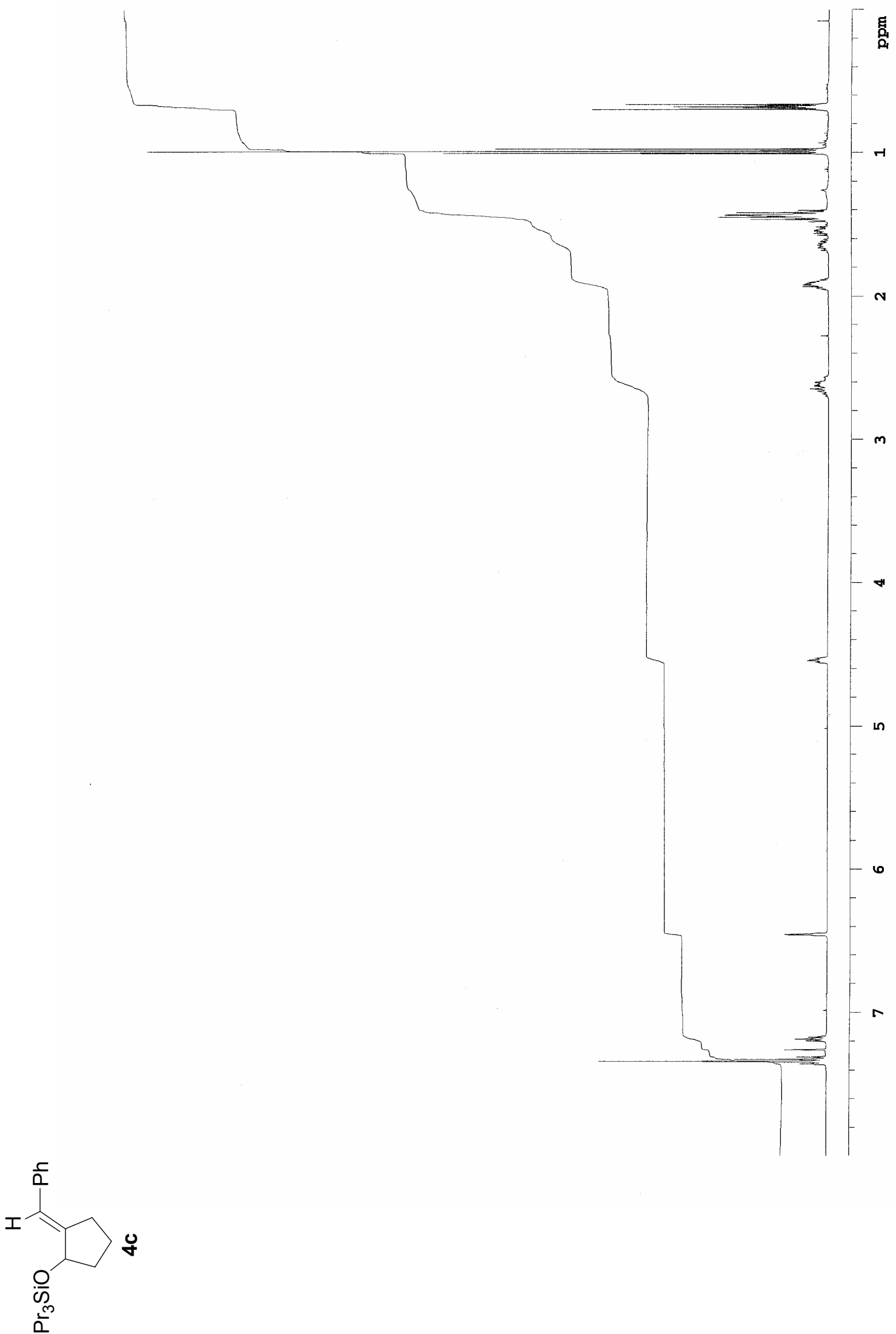

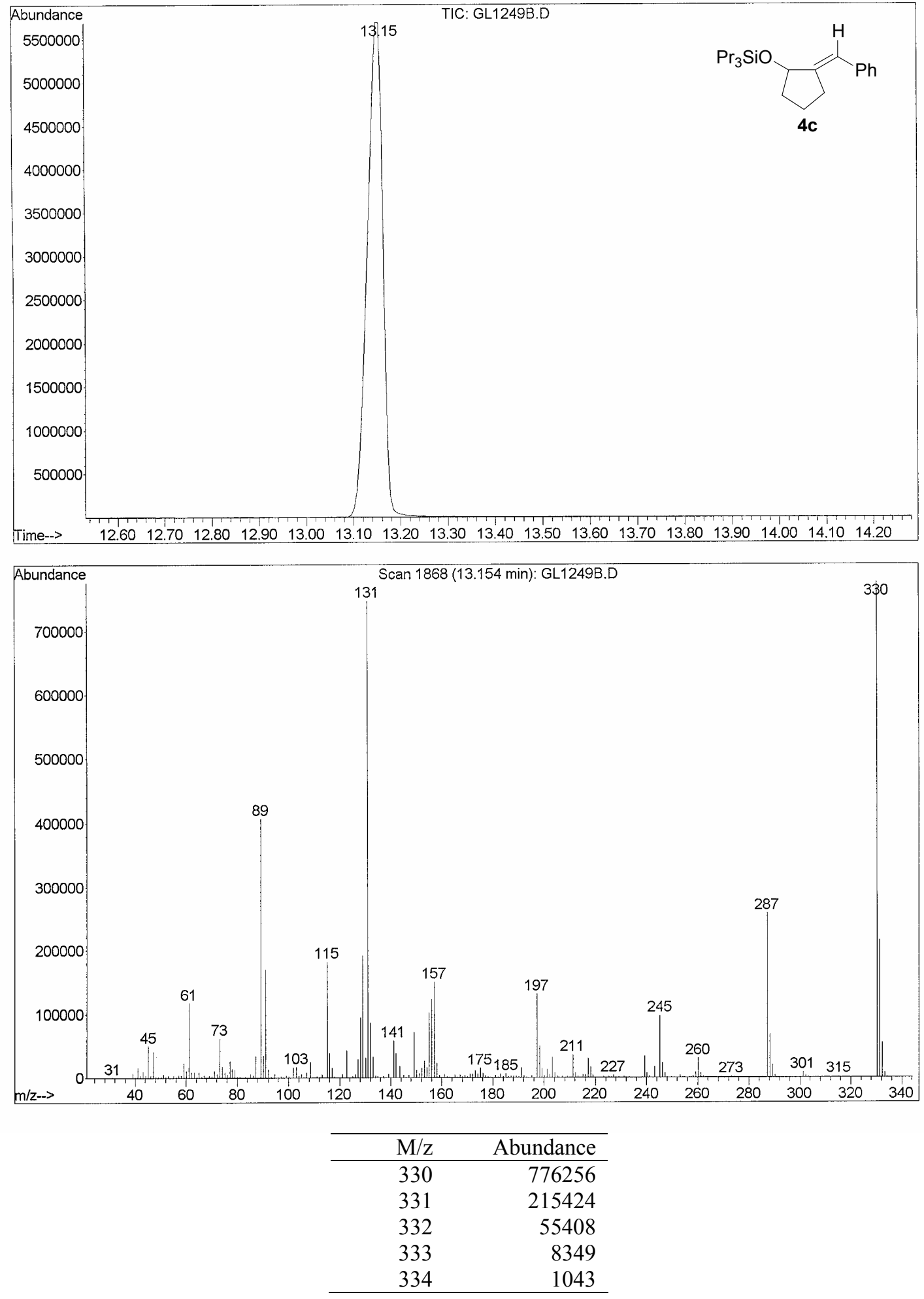


\section{Crossover experiment of $\mathrm{Ni}(\mathrm{COD})_{2}$ / carbene catalyzed cyclization of ynals:}

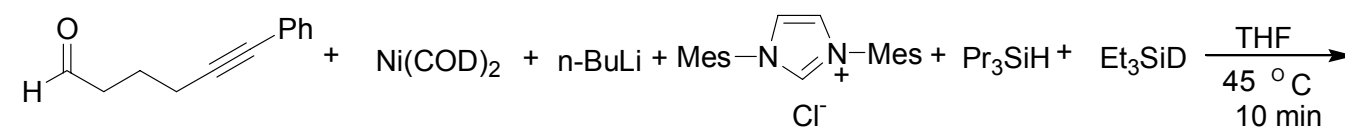<smiles>CCOC1CCCC1=Cc1ccccc1</smiles>

$4 a$

$<2 \%$<smiles>[2H]/C(=C1\CCCC1O[Si]C)c1ccccc1</smiles>

4b

$55 \%$

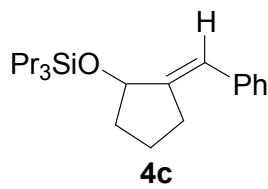

$41 \%$

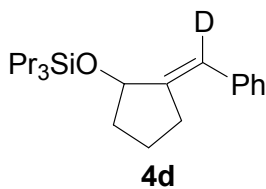

$<2 \%$

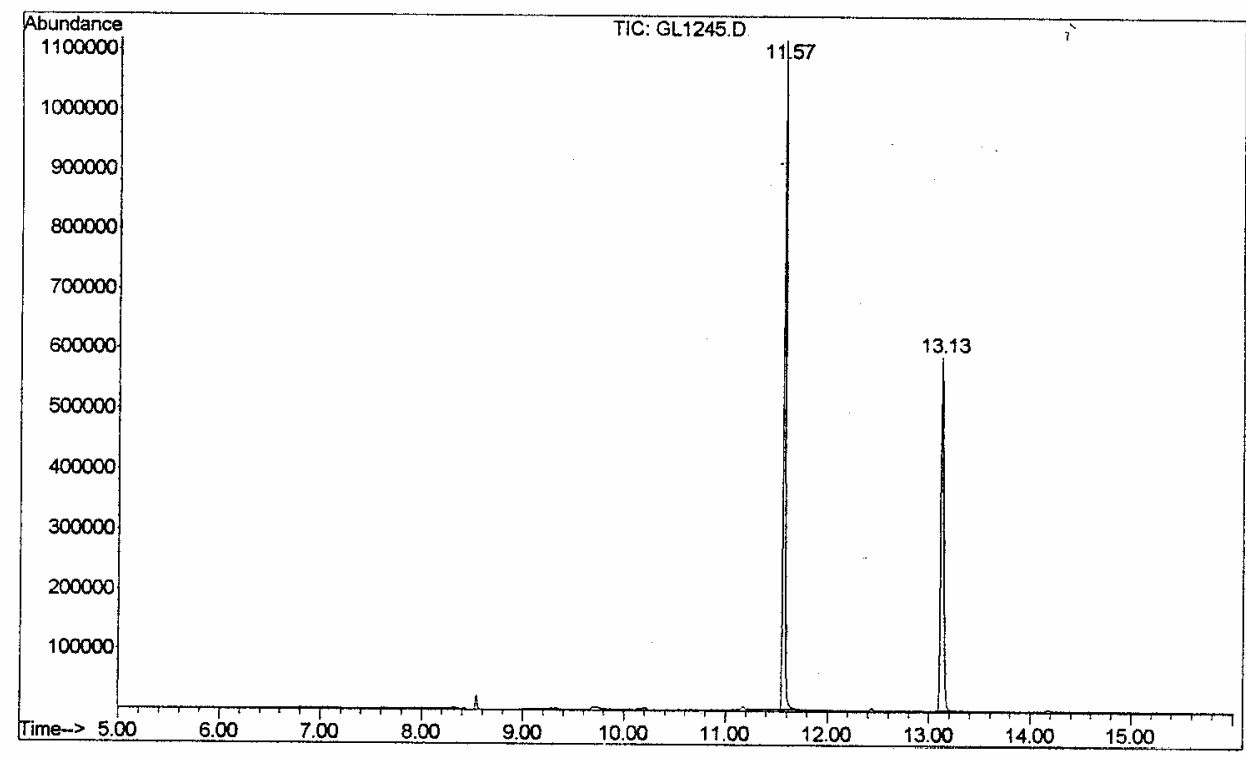

\begin{tabular}{|c|c|c|c|c|c|c|c|c|c|}
\hline $\begin{array}{c}\text { peak } \\
\#\end{array}$ & $\begin{array}{l}\text { R.T. } \\
\text { min }\end{array}$ & $\begin{array}{r}\text { first } \\
\text { scan }\end{array}$ & $\begin{array}{r}\max \\
\text { scan }\end{array}$ & $\begin{array}{l}\text { last } \\
\text { scan }\end{array}$ & $\begin{array}{l}\text { PK } \\
\text { TY }\end{array}$ & $\begin{array}{c}\text { peak } \\
\text { height }\end{array}$ & $\begin{array}{l}\text { corr. } \\
\text { area }\end{array}$ & $\begin{array}{l}\text { corr. } \\
\frac{8}{8} \max .\end{array}$ & $\begin{array}{l}\& \text { of } \\
\text { total }\end{array}$ \\
\hline-- & & & & & --- & & $\cdots \cdots$ & 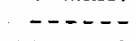 & \\
\hline $\begin{array}{l}1 \\
2\end{array}$ & $\begin{array}{l}11.571 \\
13.131\end{array}$ & $\begin{array}{l}1594 \\
1851\end{array}$ & $\begin{array}{l}1601 \\
1864\end{array}$ & $\begin{array}{l}1626 \\
1882\end{array}$ & $\begin{array}{l}\mathrm{BB} \\
\mathrm{BB}\end{array}$ & $\begin{array}{r}1070471 \\
584802\end{array}$ & $\begin{array}{l}15408799 \\
11699063\end{array}$ & $\begin{array}{r}100.00 \% \\
75.928\end{array}$ & 43 \\
\hline
\end{tabular}



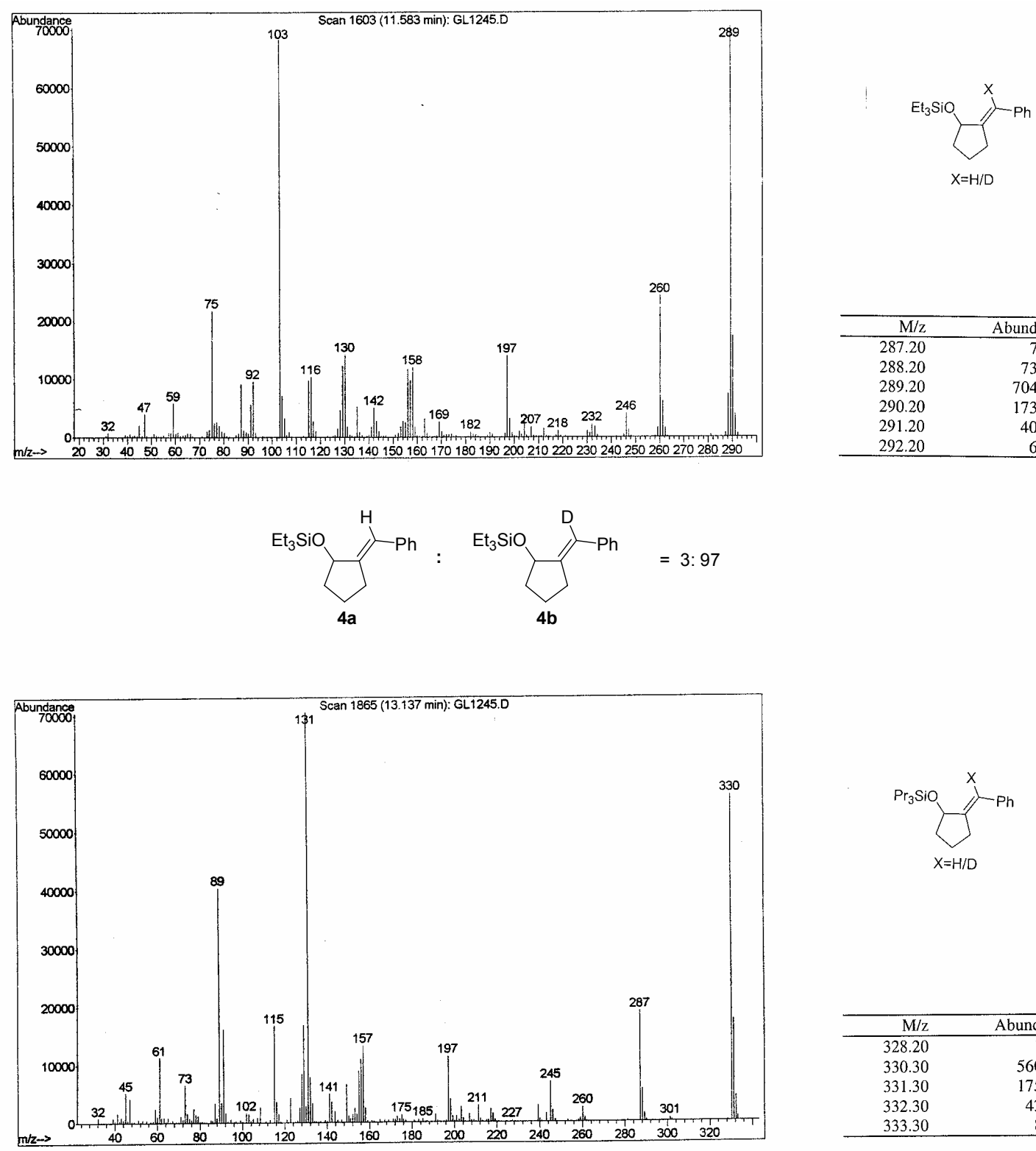

\begin{tabular}{rr}
\hline $\mathrm{M} / \mathrm{z}$ & Abundance \\
\hline 287.20 & 789.0 \\
288.20 & 7317.0 \\
289.20 & 70416.0 \\
290.20 & 17352.0 \\
291.20 & 4001.0 \\
292.20 & 625.0 \\
\hline
\end{tabular}

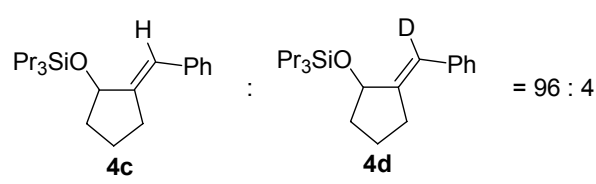

\begin{tabular}{rr}
\hline $\mathrm{M} / \mathrm{z}$ & Abundance \\
\hline 328.20 & 194.0 \\
330.30 & 56040.0 \\
331.30 & 17544.0 \\
332.30 & 4356.0 \\
333.30 & 863.0 \\
\hline
\end{tabular}




\section{Crossover experiment of $\mathrm{Ni}(\mathrm{COD})_{2} / \mathrm{PBu}_{3}$ catalyzed cyclization of ynals:}

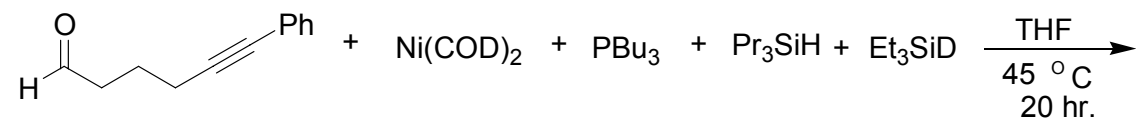

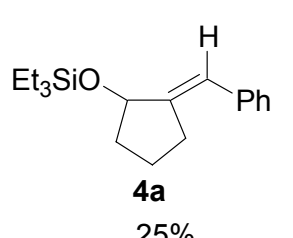

$25 \%$

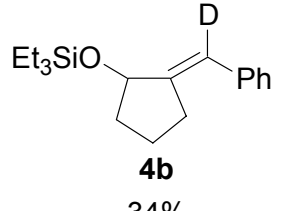

$34 \%$

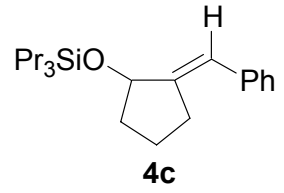

$23 \%$

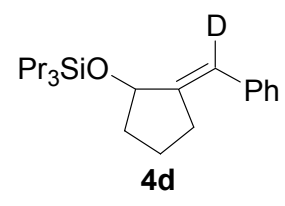

$18 \%$

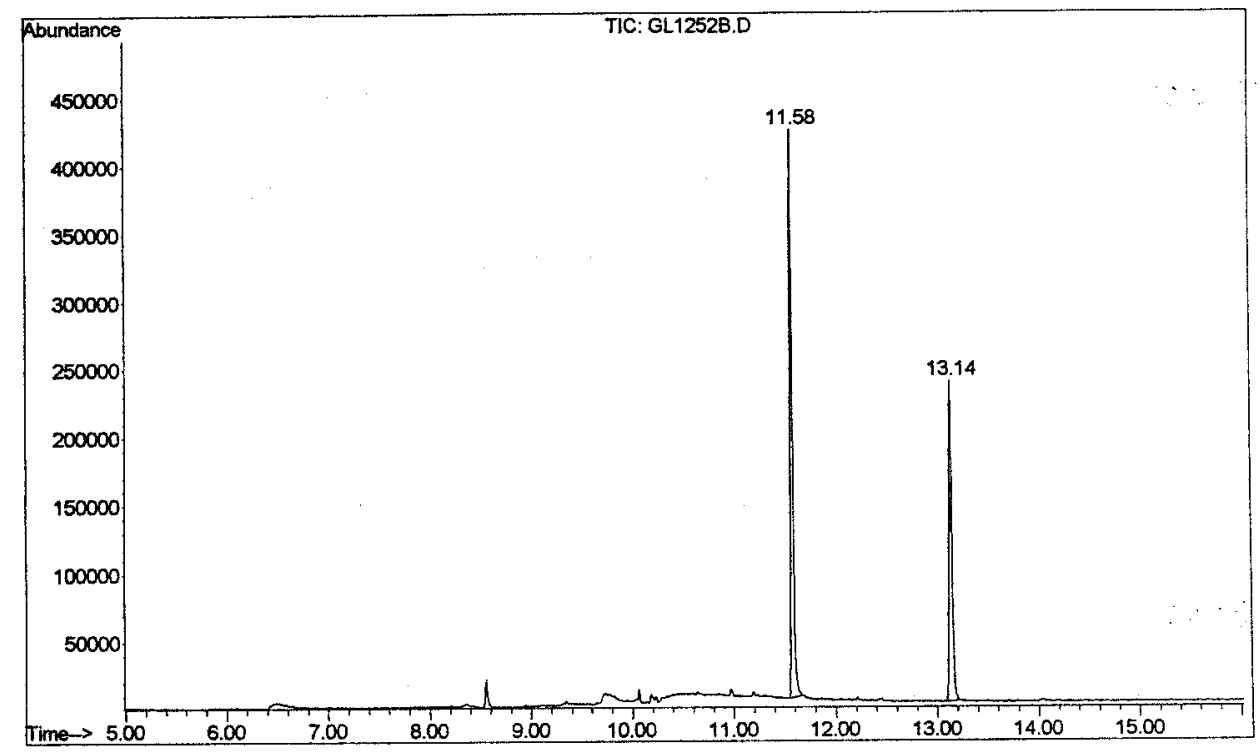

\begin{tabular}{|c|c|c|c|c|c|c|c|c|c|}
\hline \# & $\begin{array}{l}\text { R.T. } \\
\min \end{array}$ & $\begin{array}{r}\text { first } \\
\text { scan }\end{array}$ & $\begin{array}{l}\max \\
\text { scan }\end{array}$ & $\begin{array}{l}\text { last } \\
\text { scan }\end{array}$ & $\begin{array}{l}\text { PK } \\
\text { TYY }\end{array}$ & $\begin{array}{c}\text { peak } \\
\text { height }\end{array}$ & $\begin{array}{l}\text { corr. } \\
\text { area }\end{array}$ & $\begin{array}{l}\operatorname{corr} . \\
8 \max .\end{array}$ & $\begin{array}{l}\text { \& of } \\
\text { total }\end{array}$ \\
\hline & & & 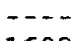 & --- & -- & ----- & ------- & ----- & \\
\hline$\frac{1}{2}$ & $\begin{array}{l}11.577 \\
13.137\end{array}$ & & $\begin{array}{l}1602 \\
1865\end{array}$ & & $\mathrm{BB}$ & & $\begin{array}{l}6464420 \\
4677618\end{array}$ & 100.0 & \\
\hline
\end{tabular}



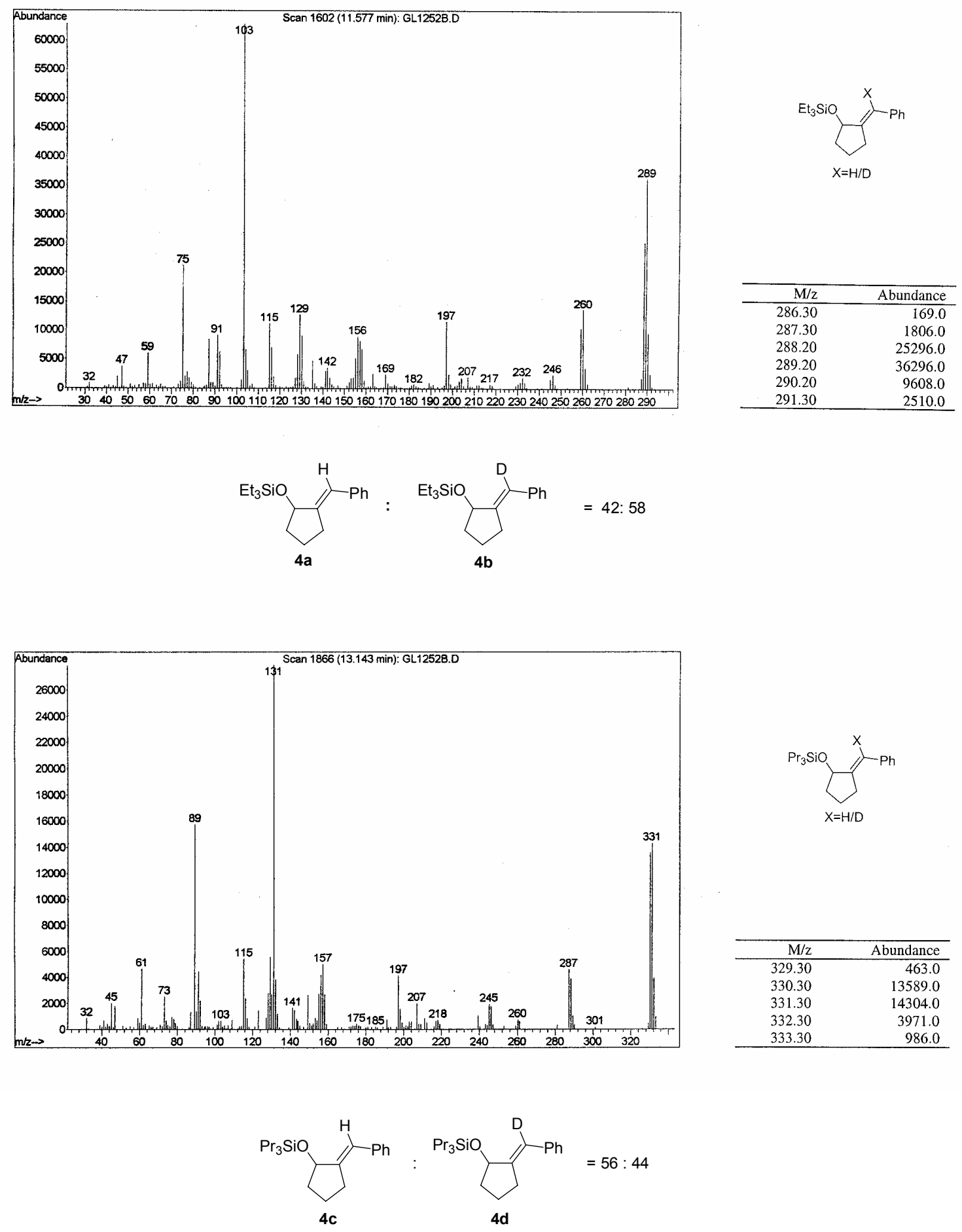